\title{
Strong Light-Matter Interactions Enabled by Polaritons in Atomically Thin Materials
}

\author{
Gonçalves, P. A. D.; Stenger, Nicolas; Cox, Joel D.; Mortensen, N. Asger; Xiao, Sanshui
}

Published in:

Advanced Optical Materials

Link to article, DOI:

10.1002/adom.201901473

Publication date:

2020

Document Version

Peer reviewed version

Link back to DTU Orbit

Citation (APA):

Gonçalves, P. A. D., Stenger, N., Cox, J. D., Mortensen, N. A., \& Xiao, S. (2020). Strong Light-Matter

Interactions Enabled by Polaritons in Atomically Thin Materials. Advanced Optical Materials, 8(5), [1901473]. https://doi.org/10.1002/adom.201901473

\section{General rights}

Copyright and moral rights for the publications made accessible in the public portal are retained by the authors and/or other copyright owners and it is a condition of accessing publications that users recognise and abide by the legal requirements associated with these rights.

- Users may download and print one copy of any publication from the public portal for the purpose of private study or research.

- You may not further distribute the material or use it for any profit-making activity or commercial gain

- You may freely distribute the URL identifying the publication in the public portal 
Abstract Atomically-thin materials have emerged as an excellent material platform for nanophotonics owing to their ability to host two-dimensional polaritons that can concentrate light on extreme-subwavelength scales. Opening with a pedagogical discussion of plasmon-polaritons in graphene, this review explores recent developments in hybrid graphene-metal nanostructures, nonlinear graphene plasmonics, and strong plasmon-exciton coupling in atomically-thin semiconductors, opening new frontiers in nano-optics.

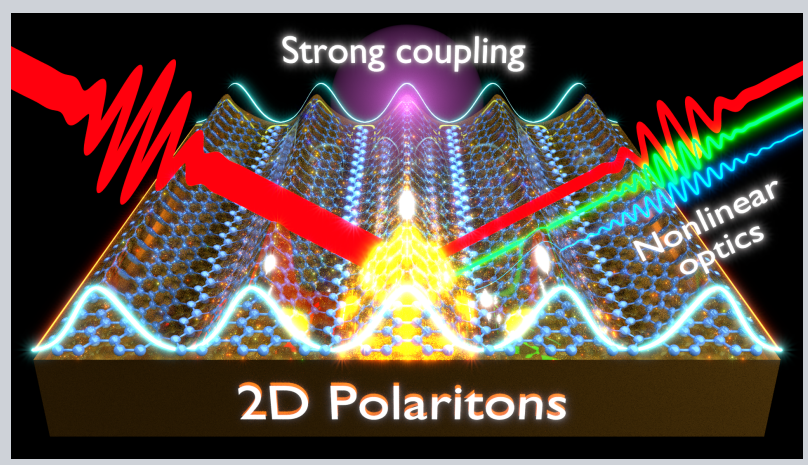

\title{
Strong Light-Matter Interactions Enabled by Polaritons in Atomically-Thin Materials
}

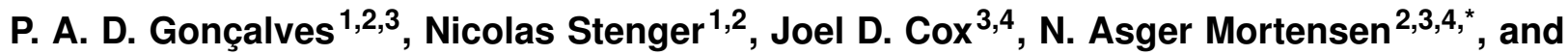 \\ Sanshui Xiao ${ }^{1,2, *}$
}

Polaritons, formed when light hybridizes with polarization charges formed at the boundaries between media with positive and negative dielectric response functions, can focus light into regions much smaller than its associated free space wavelength for a plethora of applications in nanophotonics, ranging from biological sensing to photocatalysis to nonlinear and quantum optics. In the two-dimensional (2D) limit represented by atomically-thin and van der Waals (vdW) materials of 2D layers bound by weak vdW attraction, polaritons are weakly-screened and thus characterized by extremely small wavelengths associated with extreme optical confinement, and furthermore can exhibit long lifetimes, electrical tunability, and extreme sensitivity to their dielectric environment, among many other desirable qualities in nano-optical device applications. This review summarizes the fundamentals of polaritons in atomically-thin materials, emphasizing plasmon and exciton polaritons, their strong light-matter interactions and nonlinear plasmonics. More specifically, the review opens with a pedagogical discussion of plasmons in extended and nanostructured graphene, providing a classical electrodynamical model in a nonretarded theoretical framework, and the ultraconfined acoustic plasmons supported by hybrid graphene-dielectric-metal structures, then nonlinear graphene plasmonics, to strong coupling in layered transition metal dichalcogenides (TMDC). These fundamental exploration of polaritonics enables many interesting applications in optoelectronics and biomedical technologies.

\section{Introduction}

Since the initial isolation in 2004(-2005) of single-layer graphene by mechanical exfoliation [1,2], a massive body of research on graphene and other atomically-thin materials beyond graphene has been growing rapidly, further catalyzed by the novel physics and also by the steady progress of nanofabrication technology. In particular, advanced fabrication techniques now enable unprecedented control over material growth, quality, and customization, facilitating both the discovery of new 2D materials and their availability: Nowadays, ultrafast epitaxial growth of large-scale singlecrystal graphene is achievable by situ modulation of the vapour-phase reactants [3-5], compositionally modulated $\mathrm{MoS}_{2}-\mathrm{MoSe}_{2}$ and $\mathrm{WS}_{2}-\mathrm{WSe}_{2}$ lateral heterostructures have been reported [6], and correlated insulator behaviour has been observed in stacked graphene sheets twisted by a magic angle of $1.1^{\circ}[7,8]$. Recent developments in vdW integration of 2D materials present alternative ways to create vdW heterostructures with unique and desirable electronic and optical properties that are unavailable in isolated 2D materials $[9,10]$. All of these advances have triggered fundamental explorations of newly developed 2D and vdW heterostructures, stimulating investigation of their potential applications in energy harvesting [11-14], photonics and optoelectronics [15-22], and biomedical technologies [23-28].

Due to their atomic-scale thickness, light-matter interactions in 2D materials are often very weak; the strong light-matter interactions in 2D and vdW heterostructures that have been observed are often fueled by polaritonic ex-

\footnotetext{
${ }^{1}$ Department of Photonics Engineering, Technical University of Denmark, DK-2800 Kongens Lyngby, Denmark 2 Center for Nanostructured Graphene, Technical University of Denmark, DK-2800 Kongens Lyngby, Denmark ${ }^{3}$ Center for Nano Optics, University of Southern Denmark, DK5230 Odense M, Denmark ${ }^{4}$ Danish Institute for Advanced Study, University of Southern Denmark, DK-5230 Odense M, Denmark

* Corresponding author: e-mail: asger@mailaps.org, e-mail: saxi@fotonik.dtu.dk
} 


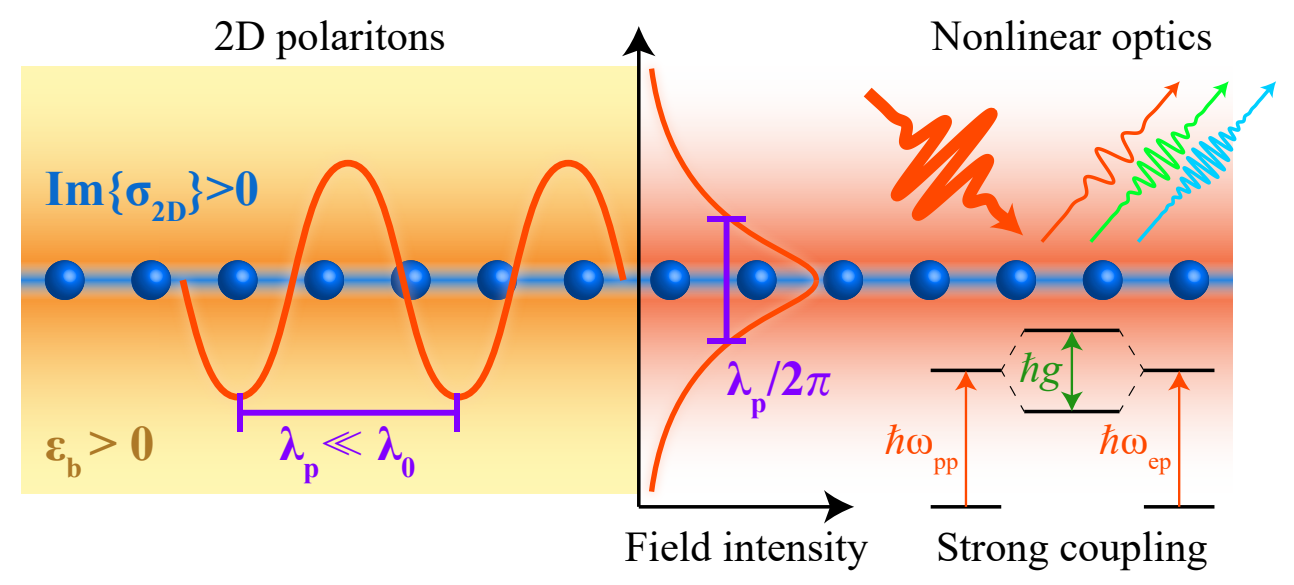

Figure 1 Schematic of polaritons in atomically-thin materials. In the two-dimensional limit, polaritons are excited in the plane of an atomically-thin crystal by light at frequencies where the imaginary part of the $2 \mathrm{D}$ optical conductivity $\sigma_{2 \mathrm{D}}$ and the dielectric permittivity of the background medium $\varepsilon_{b}$ are both positive. The polariton wavelength $\lambda_{p}$ is much smaller than the impinging light wavelength $\lambda_{0}$, with the associated electric field confined within a distance $\sim \lambda_{\mathrm{p}} / 2 \pi$ of the film; this concentrated near-field drives nonlinear optical processes (e.g., harmonic generation) and can enable strong coupling with proximal quantum emitters.

citations [29-34]. These polaritonic waves normally have extremely small in-plane wavelengths $\lambda_{\mathrm{p}}$ compared to their associated free-space light wavelength $\lambda_{0}$ (see Fig. 1). For example, the light confinement factor $\lambda_{0} / \lambda_{\mathrm{p}}$ can reach $\sim 50$ for hyperbolic phonon polariton [35-37], and even $\sim 220$ for plasmon polaritons in high-quality graphene encapsulated by hexagonal boron nitride $(\mathrm{hBN})$ and in graphene moiré superlattices [38,39]. The strong light concentration associated with $2 \mathrm{D}$ polaritons gives rise to an extremely large local density of electromagnetic states, resulting in strong light-matter interactions. Very recently, the anisotropic polariton propagation in a natural $\mathrm{vdW}$ material $\alpha-\mathrm{MoO}_{3}$ has been reported [40] to exhibit ultra low loss, ten times smaller than that of graphene plasmon polaritons at room temperature [38], strong optical Ofield confinement, with $\lambda_{o} / \lambda_{\mathrm{p}} \approx 60$. The realization of actively-tunable and long-lived polaritons in $2 \mathrm{D}$ and $\mathrm{vdW}$ materials presents a new frontier in nanophotonics to control light on length scales well below the diffraction limits. This review presents an overview of the fundamentals of polaritonic physics in extended and structured 2D materials, with an emphasis on plasmon and exciton polaritons, and their strong lightmatter interactions.

\section{Graphene plasmon polaritons}

The most prominent example of a 2D polariton in the literature is unarguably the graphene plasmon polariton [30, 41,42]. Doped graphene supports plasmon polaritons across the terahertz (THz) and mid-infrared (mid-IR) spectral ranges and it is often regarded as an excellent plasmonic material [30, 38, 41-44]. Some of the enticing properties of graphene plasmons [30, 41, 42] are consequence of graphene's tantalizing electronic and optical properties [41,45], while others are simply related to its twodimensionality $[41,46]$. Nonetheless, the unique electronic structure of graphene is of paramount importance for many of its alluring polaritonic features [32,41,42]. In addition, the mechanical stability of graphene [47] together with its intrinsic atomic thickness makes it particularly appealing also from a technological standpoint [48].

A key advantage of graphene plasmons-particularly when compared with traditional surface plasmons supported by three-dimensional (3D) metals-is the ability to actively control the frequency of plasmon resonances in graphene simply by tuning its Fermi level [49], which may be achieved by means of electrostatic gating [50-52] or by chemical doping [53]. Another noteworthy advantage of graphene plasmon polaritons is that they can propagate while experiencing relatively low losses when compared with traditional plasmonic materials (this is particularly true for graphene encapsulated in $\mathrm{hBN}$ ) [30,38,43].

In the following, we present an overview of the elementary physics governing plasmonic excitations in graphene. We begin by considering propagating plasmon polaritons supported by an extended, continuous graphene sheet, and then move on to the theoretical description of localized plasmons in graphene nanostructures. Finally, it should be noted that although here particular emphasis is given to plasmons in graphene, the theoretical methodology introduced in this section can be straightforwardly applied to other polaritonic excitations [29,30] in the ever-increasing number [54] of 2D and quasi-2D materials $[55,56]$.

\subsection{Plasmons in extended graphene}

We consider a graphene monolayer sandwiched between two dielectric media, characterized by the relative permittivities $\varepsilon_{1}$ and $\varepsilon_{2}$. The dispersion relation of transverse magnetic (TM) plasmon polaritons propagating along the graphene sheet is straightforwardly derived by matching the fields on either side of the interface [41], similarly to the derivation of the dispersion of surface plasmon polari- 


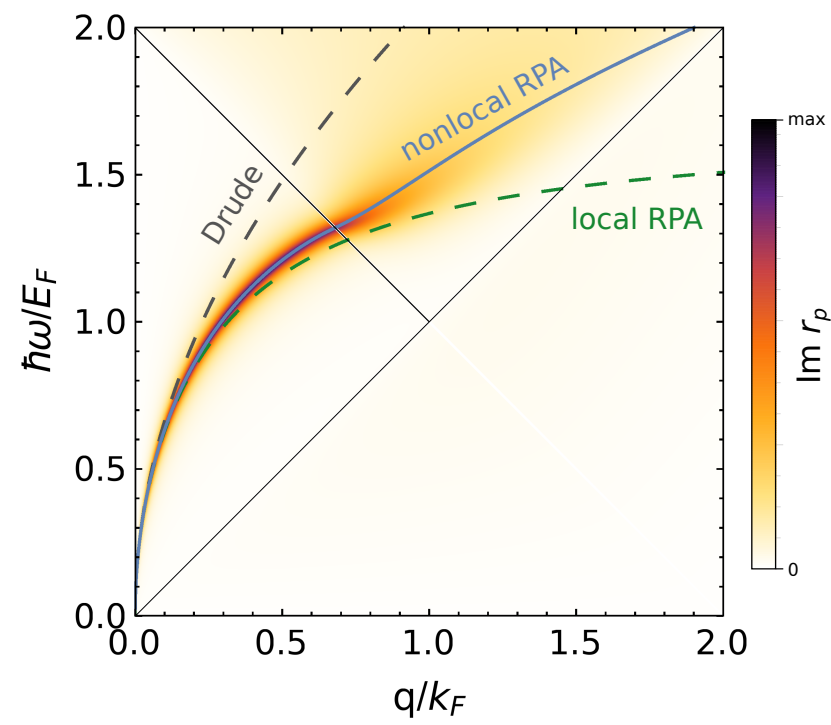

Figure 2 Dispersion relation of graphene plasmons in extended graphene. The different curves represent the graphene plasmon's dispersion [cf. Eq. (1)], here plotted as $\hbar \omega$ vs $\operatorname{Re} q$, calculated using different models for the conductivity of graphene [41] (in the zero-temperature limit), namely, the Drude-type conductivity (gray dashed line), the local RPA result (green dashed line), and the full nonlocal RPA conductivity of graphene (blue solid line) in the relaxation-time approximation using Mermin's prescription [41, 57]. The colormap in the background (here in logarithmic scale) expresses the loss function via the imaginary part of the system's reflection coefficient for $p$-polarized waves, $\operatorname{Im} r_{p}$, obtained within the full nonlocal RPA framework. Material parameters: $\varepsilon_{1}=\varepsilon_{2}=1$, $E_{\mathrm{F}}=0.4 \mathrm{eV}$, and $\hbar \gamma=8 \mathrm{meV}$.

tons (SPPs) in dielectric-metal interfaces. The crucial difference here is that the presence of graphene is taken into account by introducing a non-vanishing surface current density, $\mathbf{J}_{2 \mathrm{D}}$, which enters in the boundary conditions. For a uniform graphene layer-i.e., unstrained, unpatterned, pristine graphene-the induced surface current due to an electric field is given by $\mathbf{J}_{2 \mathrm{D}}=\sigma \mathbf{E}_{\|}$, where $\sigma \equiv \sigma(q, \omega)$ is the (in general nonlocal) conductivity of graphene [41], and $\mathbf{E}_{\|}$is the total in-plane electric field evaluated at the position of the graphene sheet. With the above-noted considerations, the dispersion relation of graphene plasmons (GPs) stems from the following implicit condition [41]:

$$
\frac{\varepsilon_{1}}{\sqrt{q^{2}-k_{0}^{2} \varepsilon_{1}}}+\frac{\varepsilon_{2}}{\sqrt{q^{2}-k_{0}^{2} \varepsilon_{2}}}+\frac{i \sigma(q, \omega)}{\omega \varepsilon_{0}}=0,
$$

where $q$ is the graphene plasmon's wavevector and $k_{0}=\omega / c$ is the wavevector of a photon with the same (angular) frequency $\omega$ in free-space. In general, this dispersion relation has to be solved by numerical means. Furthermore, from Eq. (1) it is clear that confined TM graphene plasmons can only exist in regions of the phase-space where $\operatorname{Im}\{\sigma\}>0$; in the long-wavelength limit [i.e., where $\sigma(q \rightarrow 0, \omega) \equiv$ $\sigma(\omega)]$, this is fulfilled for energies $\hbar \omega / E_{\mathrm{F}} \lesssim 1.667$ [41].
The dispersion relation of plasmons in (doped) graphene is shown in Fig. 2, where it has been calculated using three different models for the conductivity of graphene. The simplest of these is the Drude-like expression for the conductivity of graphene $[41,58-60]$, which predicts a squareroot-type dispersion, $\omega_{\mathrm{GP}} \propto \sqrt{q}$, for graphene plasmons. The next step in the complexity ladder is the inclusion of both intraband and interband contributions for graphene's conductivity within the framework of the local-response approximation (LRA), i.e., the $q \rightarrow 0$ limit, and the randomphase approximation (RPA) $[41,59,60]$, hereafter dubbed as local RPA. Lastly, we also show the dispersion of GPs obtained by employing the more sophisticated full nonlocal conductivity of graphene obtained within the formalism of the nonlocal RPA $[41,61,62]$ together with a particleconserving relaxation-time approximation $[41,57]$. The nonlocal model imparts significant deviations of the GPs' dispersion from its local prediction. Naturally, this deviation is more pronounced for large wavevectors (and correspondingly larger frequencies). Another important feature that can only be rigorously described using the full nonlocal model is the substantial change of the GPs' dispersion when it enters the region associated with interband Landau damping ( $\left.\hbar \omega>\hbar v_{\mathrm{F}} q \wedge \hbar \omega>2 E_{\mathrm{F}}-\hbar v_{\mathrm{F}} q\right)$, where GPs become short-lived due to their ability to decay rapidly into electronhole pairs [41,42]. The influence of this mechanism can be clearly inferred from the disappearance of a well defined plasmon branch in the color plot of Fig. 2, immediately after entering the interband electron-hole continuum.

Moreover, owing to the extreme subwavelength confinement promoted by GPs [38, 41-43], the nonretarded limit (defined by the absence of retardation effects) can be taken without any loss of accuracy in nearly all the relevant scenarios. In fact, treating plasmons in graphene within a nonretarded approach often constitutes an excellent approximation, as demonstrated in a number of works [38, 41-43, 63-67]. In this vein, we take the nonretarded limit (i.e., $q \gg k_{0}$ ) of Eq. (1), thereby obtaining the nonretarded dispersion relation of graphene plasmons

$$
q=\frac{2 i \omega \varepsilon_{0} \bar{\varepsilon}}{\sigma(q, \omega)},
$$

where we have introduced the quantity $\bar{\varepsilon}=\left(\varepsilon_{1}+\varepsilon_{2}\right) / 2$ without loss of generality [41]. We stress that, in general, Eq. (2) remains an implicit condition for the GPs' nonretarded spectrum, due to the explicit dependence of the conductivity on both $q$ and $\omega$. In the local limit, and for low-loss, one can obtain a closed-form expression for the (real part) of the GPs' wavevector as a function of frequency, that is, $q=\frac{2 \omega \varepsilon_{0} \bar{\varepsilon}}{\operatorname{Im} \sigma(\omega)}$ At this point, it is instructive to carry out an explicit calculation by adopting the Drude-like expression for the conductivity of graphene, i.e., $\sigma_{\mathrm{D}}(\omega)=\frac{i e^{2}}{\pi \hbar} \frac{E_{\mathrm{F}}}{\hbar \omega+i \hbar \gamma}$ [41]: within these approximations, the dispersion for graphene plasmons in extended graphene becomes (for low-loss) [41]

$$
\omega_{\mathrm{GP}}=\frac{1}{\hbar} \sqrt{\frac{2 \alpha}{\bar{\varepsilon}} E_{\mathrm{F}} \hbar c q}-i \frac{\gamma}{2},
$$


where $\alpha=\frac{e^{2}}{4 \pi \varepsilon_{0} \hbar c} \simeq 1 / 137$ denotes the fine-structure constant. Chiefly, this result predicts that plasmons in graphene exhibit a $\omega_{\mathrm{GP}} \propto \sqrt{q}$ dispersion in the local, intrabanddominated conductivity regime. Incidentally, such squareroot dependence of the plasmon dispersion with wavevector is a manifestation of the system's two-dimensionality, as this behavior is also observed in conventional $2 \mathrm{D}$ electron gases with a parabolic band $[41,46,68]$. However, the dependence of the plasmon energy with the carrier density is distinct among graphene and traditional two-dimensional electron gas (2DEG) in semiconductor heterostructures. In particular, graphene plasmon's exhibit a $\omega_{\mathrm{GP}} \propto \sqrt{q} n_{e}^{1 / 4}$ dependence, whereas plasmons in a conventional 2DEG exhibit a $\omega_{\text {plasmon }}^{2 \mathrm{DEG}} \propto \sqrt{q} n_{e}^{1 / 2}$ scaling instead $^{1}$ [41]. Evidently, this difference can be ascribed to the linear energy-momentum dispersion that is characteristic of graphene's massless Dirac fermions.

Before moving on to the discussion of plasmons in graphene nanostructures, it is instructive to obtain a rough estimate of the maximum confinement of the electromagnetic field that can be achieved using plasmons in extended, continuous single-layer graphene. From Eq. (3), the ratio between the GP's wavelength, $\lambda_{\mathrm{GP}}=2 \pi / q_{\mathrm{GP}}$, and that of photon in free-space, $\lambda_{0}=2 \pi c / \omega$, with the same frequency $\omega$, is found to be [41]

$$
\frac{\lambda_{\mathrm{GP}}}{\lambda_{0}}=\frac{2 \alpha}{\bar{\varepsilon}} \frac{E_{\mathrm{F}}}{\hbar \omega} .
$$

For graphene on a typical dielectric substrate, and for frequencies near $\omega \approx E_{\mathrm{F}} / \hbar$ (i.e., sufficiently away from interband effects), which typically falls in the mid-IR, one obtains $\lambda_{\mathrm{GP}} \approx \alpha \lambda_{0} \sim 10^{-2} \lambda_{0}$, that is, the GP's wavelength can be up to 100 times smaller than that of light propagating in free-space.

\footnotetext{
1 As detailed in the text, the nonretarded dispersion relation for plasmons supported by $2 \mathrm{D}$ electron systems can be generically written as $q=2 \omega \varepsilon_{0} \bar{\varepsilon} / \operatorname{Im} \sigma(\omega)$, assuming negligible loss. Hence, for a Drude-type 2D conductivity, this translates to

$$
\omega_{2 \text { D-plasmon }}=\sqrt{\frac{\mathscr{D}}{2 \pi \varepsilon_{0} \bar{\varepsilon}} q},
$$

where $\mathscr{D}$ is the so-called Drude weight. This quantity is defined via the Drude conductivity for an arbitrary 2D electron system, i.e., $\sigma(\omega)=\frac{i}{\pi} \frac{\mathscr{D}}{\omega+i \gamma}$. Since the Drude weight of graphene, $\mathscr{D}_{\mathrm{G}}=e^{2} E_{\mathrm{F}} / \hbar^{2}=e^{2} v_{F} \sqrt{\pi n_{e}} / \hbar$, is different than that of a parabolic 2DEG, $\mathscr{D}_{2 \mathrm{DEG}}=e^{2} \pi n_{e} / \mathrm{m}^{*}$, this leads to $2 \mathrm{D}$ plasmons whose dispersions exhibit different dependencies as a function of the electronic density, namely
}

$$
\omega_{2 \mathrm{D}-\text { plasmon }}=\left\{\begin{array}{ll}
C_{\mathrm{G}} q^{1 / 2} n_{e}^{1 / 4} & , \text { for plasmons in graphene } \\
C_{2 \mathrm{DEG}} q^{1 / 2} n_{e}^{1 / 2} & , \text { for plasmons in a 2DEG }
\end{array},\right.
$$

where, for the sake of completeness, the constants in the previous expressions are $C_{\mathrm{G}}=\sqrt{\frac{e^{2}}{2 \sqrt{\pi} \varepsilon_{0} \bar{\varepsilon}} v_{F}}$ and $C_{2 \mathrm{DEG}}=\sqrt{\frac{e^{2}}{2 \varepsilon_{0} \bar{\varepsilon} m^{*}}}$.

\subsection{Plasmons in nanostructured graphene and other $2 D$ nanostructures}

The studies carried out in Sect. 2.1 on the basic properties of propagating graphene plasmons in extended graphene systems have enabled us to establish the fundamental theory and the elementary concepts underpinning plasmonic excitations in graphene. Notwithstanding, the vibrant field of graphene plasmonics $[32,41,42]$ is considerably wealthier, going well beyond the restricted subgroup of infinite, extended graphene structures. Below, we shall expand our previous considerations by discussing localized plasmons in graphene nanostructures $[41,66]$. These are typically fabricated by patterning an otherwise continuous graphene sheet, and arguably constitute the most abundant subset of graphene-based plasmonic structures considered in the scientific literature. A primary reason for this has to do with the fact that plasmons in nanostructured graphene can couple to light directly without the necessity to employ special coupling techniques (a feature that they share with LSPs in finite-sized metal nanoparticles). Examples of graphene nanostructures-considered either individually or in periodic arrangements - that have been used in plasmonics are graphene ribbons [23, 50, 69-76], disks [77-84], rings $[78,80]$, triangles $[85,86]$, or anti-dots $[82,87,88]$, just to mention a few.

\subsubsection{Nonretarded theoretical framework for $2 \mathrm{D}$ polaritons in generic 2D nanostructures}

The deeply subwavelength character of graphene plasmons renders retardation effects negligible, and therefore a nonretarded treatment provides an excellent description of plasmons in graphene nanostructures in virtually all situations. Consequently, instead of solving the full vectorial Maxwell's equations, one can afford to solve the corresponding electrostatic problem, whereby plasmon excitations are governed by the Poisson equation for the (scalar) electric potential, i.e., $\nabla^{2} \Phi(\mathbf{r})=-\frac{\rho(\mathbf{r})}{\varepsilon_{0} \bar{\varepsilon}}$. Both the electrostatic potential and the charge density are herein assumed to have a harmonic time dependence of the form $\Phi(\mathbf{r}, t)=\Phi(\mathbf{r}) e^{-i \omega t}$ and $\rho(\mathbf{r}, t)=\rho(\mathbf{r}) e^{-i \omega t}$. Additionally, the charge density is restricted to the $2 \mathrm{D}$ material's plane, say, the $x y$-plane, and thus one may write $\rho(\mathbf{r})=\rho_{2 \mathrm{D}}\left(\mathbf{r}_{\|}\right) \delta(z)$, where $\rho_{2 \mathrm{D}}$ is a surface charge density and the in-plane vector $\mathbf{r}_{\|}$is given by $\mathbf{r}_{\|}=x \hat{\mathbf{x}}+y \hat{\mathbf{y}}$. Furthermore, the induced surface charge density can be written in terms of the in-plane electrostatic potential $\phi\left(\mathbf{r}_{\|}\right)$[with $\phi\left(\mathbf{r}_{\|}\right) \equiv \Phi\left(\mathbf{r}_{\|}, z=0\right)$ ] by combining Ohm's law together with the continuity equation, yielding the following relation $\rho_{2 \mathrm{D}}\left(\mathbf{r}_{\|}\right)=i \omega^{-1} \sigma(\omega) \boldsymbol{\nabla}_{\|} \cdot\left[f\left(\mathbf{r}_{\|}\right) \boldsymbol{\nabla}_{\|} \phi\left(\mathbf{r}_{\|}\right)\right]$where $\boldsymbol{\nabla}_{\|} \equiv$ $\hat{\mathbf{x}} \frac{\partial}{\partial x}+\hat{\mathbf{y}} \frac{\partial}{\partial y}$ is the in-plane 2D nabla operator and $f\left(\mathbf{r}_{\|}\right)$is an envelope function that takes into account the geometry of the 2D nanostructure $[41,42]$. Specifically, it can be defined through $\sigma\left(\mathbf{r}_{\|}, \omega\right) \equiv \sigma(\omega) f\left(\mathbf{r}_{\|}\right)$, where $f\left(\mathbf{r}_{\|}\right)=1$ for $\mathbf{r}_{\|}$within 2D nanostructure and $f\left(\mathbf{r}_{\|}\right)=0$ otherwise. Hence, the nonretarded optical response can be determined in a 
self-consistent fashion by solving Poisson equation together with above-mentioned equation that relates the charge density $\rho_{2 \mathrm{D}}\left(\mathbf{r}_{\|}\right)$with the in-plane potential $\phi\left(\mathbf{r}_{\|}\right)$. We can make this more clear by considering Poisson's equation in integral form instead, namely

$\Phi(\mathbf{r})=\frac{i \sigma(\omega)}{\omega \varepsilon_{0} \bar{\varepsilon}} \int d \mathbf{r}_{\|}^{\prime} G\left(\mathbf{r}_{\|}, \mathbf{r}_{\|}^{\prime} ; z, 0\right)\left\{\nabla_{\|}^{\prime} \cdot\left[f\left(\mathbf{r}_{\|}^{\prime}\right) \nabla_{\|}^{\prime} \phi\left(\mathbf{r}_{\|}^{\prime}\right)\right]\right\}$,

where $G\left(\mathbf{r}, \mathbf{r}^{\prime}\right)$ is the Green's function defined via $\nabla^{2} G\left(\mathbf{r}, \mathbf{r}^{\prime}\right)=$ $-\delta\left(\mathbf{r}-\mathbf{r}^{\prime}\right)$. Crucially, notice that the Eq. (5) tells us that the potential in the entire space can be calculated provided that the in-plane potential within the 2D nanostructure is known [this is the only contribution since outside the material $f\left(\mathbf{r}_{\|}\right)=0$ ]. To that end, we now evaluate the previous equality at $z=0$, thereby obtaining the ensuing self-consistent integro-differential equation for the potential within the $2 \mathrm{D}$ nanostructure:

$$
\phi\left(\mathbf{r}_{\|}\right)=\frac{i \sigma(\omega)}{\omega \varepsilon_{0} \bar{\varepsilon}} \int d \mathbf{r}_{\|}^{\prime} g\left(\mathbf{r}_{\|}, \mathbf{r}_{\|}^{\prime}\right)\left\{\nabla_{\|}^{\prime} \cdot\left[f\left(\mathbf{r}_{\|}^{\prime}\right) \nabla_{\|}^{\prime} \phi\left(\mathbf{r}_{\|}^{\prime}\right)\right]\right\},
$$

with $g\left(\mathbf{r}_{\|}, \mathbf{r}_{\|}^{\prime}\right) \equiv G\left(\mathbf{r}_{\|}, \mathbf{r}_{\|}^{\prime} ; z=0, z^{\prime}=0\right)$. Equation (6) becomes particularly elucidative (and useful) when written in terms of dimensionless variable: introducing $\tilde{\mathbf{r}}_{\|}=\mathbf{r}_{\|} / L$ and $\tilde{\nabla}_{\|}=L \boldsymbol{\nabla}_{\|}$, where $L$ is some appropriate characteristic length-scale of the structure under consideration, the above equation can be recast as

$$
\Lambda \phi\left(\tilde{\mathbf{r}}_{\|}\right)=-2 \int d \tilde{\mathbf{r}}_{\|}^{\prime} g\left(\tilde{\mathbf{r}}_{\|}, \tilde{\mathbf{r}}_{\|}^{\prime}\right)\left\{\tilde{\nabla}_{\|}^{\prime} \cdot\left[f\left(\tilde{\mathbf{r}}_{\|}^{\prime}\right) \tilde{\nabla}_{\|}^{\prime} \phi\left(\tilde{\mathbf{r}}_{\|}^{\prime}\right)\right]\right\}
$$

$$
\text { where } \Lambda=\frac{2 i \omega \varepsilon_{0} \bar{\varepsilon} L}{\sigma(\omega)}
$$

which is now clearly scale-invariant. This is because the integral has been stripped of any reference to the structure's size upon introducing the above-noted dimensionless spatial variables. Moreover, there is no frequency or any material dependencies left in the right-hand side of Eq. (7a), and thus that term has a purely geometrical meaning. Consequently, this formalism can in fact be applied to any $2 D$ material that is capable of supporting either plasmon polariton or other collective modes, e.g., phonon polaritons or exciton polaritons.

Naturally, the usefulness of the general scale-independent integro-differential equation epitomized by Eq. (7a) hinges upon one's ability to solve it. In general, this cannot be done analytically and therefore one has to rely on numerical methods. In spite of this, it is still possible to solve Eq. (7a) using semi-analytical techniques [41,66]. Broadly speaking, the idea behind such semi-analytical methods is to expand the electric potential $\phi\left(\tilde{\mathbf{r}}_{\|}\right)$using a suitable set of basis functions. Here, we have chosen to base our semi-analytical framework on an expansion using a basis containing orthogonal polynomials [41, 63, 65, 89-94]. The specific type of orthogonal polynomials [95] is intimately related to the particular geometry of the nanostructure under consideration (see, for instance, Ref. [41]). In any case, by performing such an expansion of the potential appearing in Eq. (7a) followed by the use of the appropriate orthogonality relation [95], the integro-differential equation (7a) can be transformed into a standard matrix eigenvalue problem

$$
\sum_{m=0}^{\infty} U_{n m}^{(v)} c_{m}^{(v)}=\Lambda_{v} c_{n}^{(v)} \Rightarrow \mathbf{U}_{v} \mathbf{c}_{v}=\Lambda_{v} \mathbf{c}_{v}
$$

where the index $v$ categorizes the polaritonic resonance. Finally, the diagonalization of the square-matrix $\mathbf{U}^{(v)}$ yields the system's eigenfrequencies (polaritonic resonances) via

$$
\Lambda_{v}\left(\omega_{v}\right)=\lambda_{v} \quad \Leftrightarrow \quad \frac{2 i \omega_{v} \varepsilon_{0} \bar{\varepsilon} L}{\sigma\left(\omega_{v}\right)}=\lambda_{v}
$$

where $\lambda_{v}$ denotes the eigenvalues of $\mathbf{U}^{(v)}$. On the other end, the corresponding eigenvectors allow the construction of the in-plane potential in the $2 \mathrm{D}$ nanostructure and thus the mode profile associated with the polaritonic eigenfrequency $\omega_{v}$. From here, one can also compute the induced charge density within the 2D material using $\rho_{2 \mathrm{D}}\left(\mathbf{r}_{\|}\right)=i \omega^{-1} \boldsymbol{\sigma}(\omega) \boldsymbol{\nabla}_{\|} \cdot\left[f\left(\mathbf{r}_{\|}\right) \boldsymbol{\nabla}_{\|} \phi\left(\mathbf{r}_{\|}\right)\right]$, or to determine the potential in the entire three-dimensional space through Eq. (5) [the corresponding electric field then simply follows from $\mathbf{E}(\mathbf{r})=-\nabla \Phi(\mathbf{r})]$. Notice that either one of those calculations do not require a significant amount of labor, since they only contain derivatives or integrals of functions involving polynomials and simple weighting functions (typically either an exponential, a Gaussian or a polynomial).

Before applying this formalism to any particular $2 \mathrm{D}$ polaritonic structure, it is enlightening to substitute graphene's Drude conductivity in Eq. (9) and thereby establish an explicit connection between the eigenfrequencies $\omega_{v}$ and the eigenvalues $\lambda_{v}$. Hence, assuming low-loss (i.e., $\omega_{v} \ll \gamma$ ), one finds the relation

$$
\omega_{v} \simeq \frac{1}{\hbar} \sqrt{\frac{2 \alpha}{\bar{\varepsilon}} E_{\mathrm{F}} \hbar c \frac{\lambda_{v}}{L}}-i \frac{\gamma}{2},
$$

which bears close resemblance to the dispersion relation of GPs in extended graphene [cf. Eq. (3)], where the role previously played by the wavevector is now transferred to $\lambda_{v} L^{-1}$. Notably, this result formally validates the intuitively expected behavior $\omega \propto \sqrt{1 / L}$, that here is now appropriately weighted by the eigenvalue $\lambda_{v}$ that accounts for the particular geometry of the graphene nanostructure.

\subsubsection{Plasmons in two-dimensional nanoribbons}

In the context of graphene plasmonics, studies using the ribbon geometry proliferate in the literature [23,41, 50, 69-74], thereby making it a prototypical system to study plasmons in two dimensions. Below, we provide an economical overview of the application of the nonretarded framework outlined above (Sect. 2.2.1) to the specific case of the ribbon geometry.

In what follows, we consider a 2D nanoribbon of finite width $W=2 a$ (along the $x$-axis) and that is translational 


\begin{tabular}{cccccc}
\hline \multicolumn{5}{c}{$\lambda_{\beta}^{[n]}=c_{1}^{n} \beta+\frac{4}{1+c_{2}^{n}+c_{3}^{n} \beta+c_{5}^{n} \beta^{2}}$} \\
\hline \hline$n$ & $c_{1}^{n}$ & $c_{2}^{n}$ & $c_{3}^{n}$ & $c_{4}^{n}$ & $c_{5}^{n}$ \\
\hline 0 & 0.8216 & 0 & -12.397 & 233.56 & -9.6187 \\
1 & 0.8106 & 1.1541 & 0.2760 & 0.5905 & 1.5169 \\
2 & 1 & 2.7402 & 0.1302 & 0.3411 & 0.1928 \\
3 & 1 & 4.2980 & 0.2383 & 0.2633 & 0.0667 \\
4 & 1 & 5.8731 & 0.2856 & 0.2069 & 0.0336 \\
5 & 1 & 7.4389 & 0.3055 & 0.1683 & 0.0200 \\
6 & 1 & 9.0120 & 0.3138 & 0.1413 & 0.0131 \\
7 & 1 & 10.579 & 0.3151 & 0.1211 & 0.0092 \\
8 & 1 & 12.152 & 0.3119 & 0.1057 & 0.0068 \\
9 & 1 & 13.732 & 0.3044 & 0.0933 & 0.0052 \\
\hline
\end{tabular}

Table 1 Fits to the eigenvalues describing the spectrum of plasmons in a $2 \mathrm{D}$ nanoribbon. Analytical fitting function (top row) written as a linear term plus a Padé approximant of the form [1/2], followed by the corresponding fitting constants obtained by fitting the analytical formula to the calculated data presented in Fig. 3.

invariant along the $y$-direction. Hence, the electric potential in such a system can be written as $\Phi(\mathbf{r})=\Phi(x, z) e^{i k_{y} y}$ [and similarly for the charge density]; thus the wavevector parallel to the ribbon's edges, $k_{y}$, can be used to parameterize the plasmon modes of the ribbon. Concretely, in the language of Eq. (7), the in-plane potential $\phi\left(\tilde{\mathbf{r}}_{\|}\right)$and the function $g\left(\tilde{\mathbf{r}}_{\|}, \tilde{\mathbf{r}}_{\|}^{\prime}\right)$ are found to be given by $\phi\left(\tilde{\mathbf{r}}_{\|}\right)=\phi(\tilde{x}) e^{i \beta \tilde{y}}$ and $g\left(\tilde{x}, \tilde{x}^{\prime}\right)=(2 \pi)^{-1} K_{0}\left(\beta\left|\tilde{x}-\tilde{x}^{\prime}\right|\right)$ [41], where $K_{0}$ is the zero-th order modified Bessel function of the second kind, and where we have introduced the dimensionless variables $(\tilde{x}, \tilde{y})=(x / a, y / a)$ and normalized wavevector $\beta=k_{y} a$. Likewise, since we have assigned $L \equiv a$ (i.e., half of the ribbon's width), the dimensionless parameter (7b) is then $\Lambda=2 i \omega \varepsilon_{0} \bar{\varepsilon} a / \sigma(\omega)$. Having specified all the ingredients entering in the integro-differential equation (7), the next step is to expand the potential $\phi(\tilde{x})$ using a suitable basis in terms of orthogonal polynomials. For the ribbon geometry, such an expansion takes the form $\phi(\tilde{x})=\sum_{n=0}^{\infty} c_{n} P_{n}(\tilde{x})$, where $P_{n}(\tilde{x})$ denotes the Legendre polynomials. Finally, substituting the aforementioned expansion into Eq. (7a) and exploiting the orthogonality relation of the Legendre polynomials [95] yields an eigenproblem in the familiar form of Eq. (8).

The plasmon spectrum of 2D nanoribbons calculated using the method described in the previous paragraph is shown in Fig. 3. As depicted in Fig. 3a, the eigenvalues $\lambda_{\beta}=\left\{\lambda_{\beta}^{[0]}, \lambda_{\beta}^{[1]}, \lambda_{\beta}^{[2]}, \ldots\right\}$ are parameterized by $\beta$ and form a manifold of plasmon bands indexed by $n$, where $n=0$ corresponds to the monopolar eigenmode, $n=1$ to the dipolar eigenmode, and so on. Each band therefore describes a ribbon plasmon propagating along the $y$-direction with (normalized) wavevector $\beta$ and exhibiting quantization (for $n \geq 1$ ) across the ribbon's width. In addition to our semianalytical results (shown as colored solid lines), we have also superimposed analytical fits (represented by the black
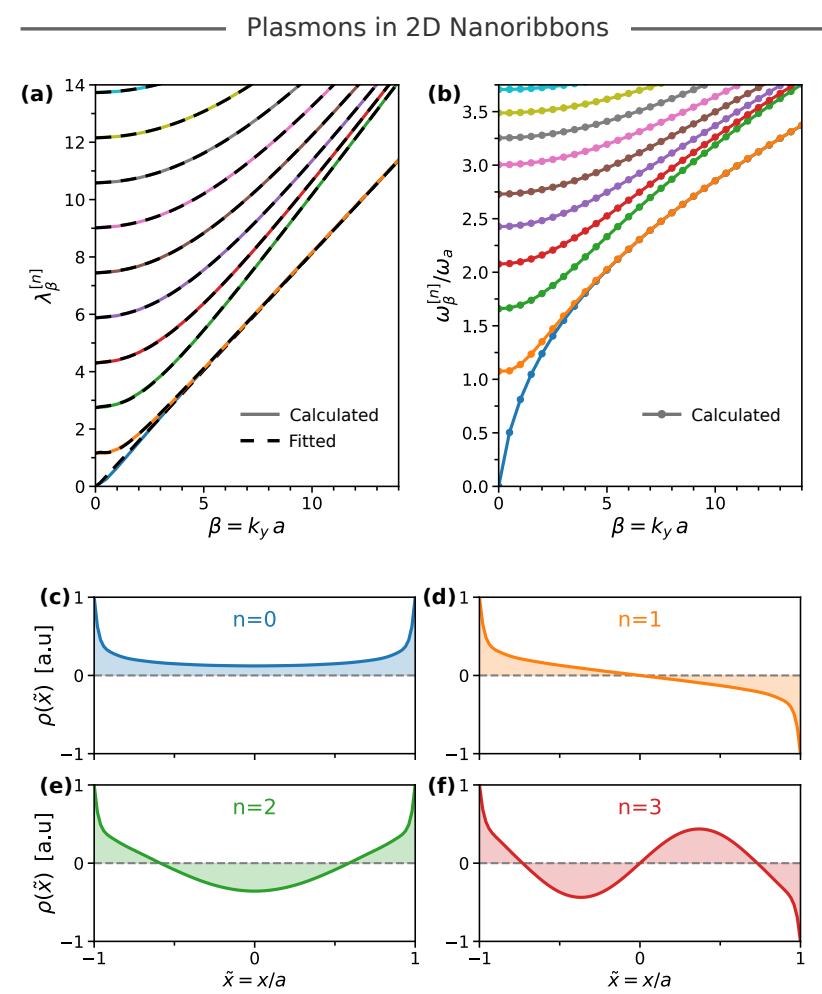

Figure 3 Plasmons in an individual 2D nanoribbon of width $W=$ 2a. (a) Eigenvalues associated with plasmons of a 2D nanoribbon calculated using the semi-analytical method described in the text (colored solid lines) and corresponding fits (black dashed lines). (b) Dispersion relation of the plasmonic eigenmodes supported by $2 \mathrm{D}$ nanoribbons, obtained using the calculated eigenvalues and assuming a Drude-type conductivity [see Eqs. (9) and (10)]. Here, we have defined $\omega_{a}=\sqrt{\frac{\mathscr{D}}{2 \pi \varepsilon_{0} \bar{\varepsilon}} a^{-1}}$, where $\mathscr{D}$ is the Drude weight of the $2 \mathrm{D}$ electron system $\left(\mathscr{D}_{\mathrm{G}}=e^{2} E_{F} / \hbar^{2}\right.$ for graphene, and $\mathscr{D}_{2 \mathrm{DEG}}=e^{2} \pi n_{e} / m^{*}$ for a conventional 2DEG). (c-f) Spatial profiles of the charge density across the ribbon associated with the four lowest-frequency plasmon eigenmodes, for a fixed $\beta=k_{y} a=1.5$. The quantities in each panel are normalized with respect to their absolute maxima. All results $[(a-f)]$ were obtained by truncating the eigensystem with $N=20$ [cf. Eq. (8)], which provides converged results for the depicted modes.

dashed lines) to the corresponding data, and whose fitting parameters are listed in Table 1. Similarly, Fig. 3b shows the same results, but where a Drude-like conductivity has been assumed, thereby allowing us to write the plasmon dispersion diagram as $\omega_{\beta}^{[n]}=\omega_{a} \sqrt{\lambda_{\beta}^{[n]}}$ [cf. Eq. (10)], where $\omega_{a}=\hbar^{-1} \sqrt{(2 \alpha / \bar{\varepsilon}) E_{F} \hbar c a^{-1}}$ for the case of a graphene nanoribbon.

Figures $3 c-f$ portray the calculated charge density profiles that are associated with the four lowest-frequency eigenmodes, and empirically endorse the standing-wave-like picture mentioned previously. Notwithstanding, in the limit of large $\beta=k_{y} a$, the plasmon bands indicated in Figs. 3a$\mathrm{b}$ can be divided into two groups based on their asymp- 
totic behavior: one containing the two lowest-frequency eigenmodes, characterized by $\lambda_{\beta}^{[n \leq 1]}$, and another containing the modes defined by $\lambda_{\beta}^{[n \geq 2]}$. In particular, the dispersion curves associated with the monopolar and dipolar ribbon eigenmodes become degenerate and asymptotically approach the dispersion of quasi-1D edge plasmons supported by a half-sheet, specifically $\lim _{\beta \rightarrow \infty} \lambda_{\beta}^{[n \leq 1]}=\lambda_{\mathrm{hs}} \beta$, where $\lambda_{\mathrm{hs}} \approx 0.8216$ (which can also be obtained using the WienerHopf technique [96]). This observation can be intuitively understood by noting that these two modes are highly - and solely - confined to the ribbon's edges. On the other hand, the dispersion curves corresponding to eigenmodes affiliated with the second group (containing the set of higher-order modes with $n \geq 2$ ) pile-up at the line defined by $\lambda_{\beta}^{[n \geq 2]}=\beta$, when $\beta \rightarrow \infty$. As such, they acquire a successively more bulk-like behavior with growing $\beta$, and their dispersion becomes $\omega_{\beta}^{[n \geq 2]} \rightarrow \omega_{k_{y}}^{[n \geq 2]}=\hbar^{-1} \sqrt{(2 \alpha / \bar{\varepsilon}) E_{F} \hbar c k_{y}}$, i.e., indistinguishable from that of plasmons in an extended, infinite graphene sheet [cf. Eq.(3)].

\subsubsection{Plasmons in two-dimensional nanodisks}

Like the ribbon, the disk geometry is also one of the most prominent nanostructures for investigating plasmons in nanostructured graphene, a fact that is reflected by the vast number of works devoted to this configuration [41,77-83]. In the following, we outline the key points for applying the nonretarded formalism introduced in this section to describe plasmon resonances supported by a conductive 2D nanodisk (e.g., a doped graphene nanodisk).

Let us consider a single 2D disk of radius $R$ made from an atomically-thin material. In the present case, by virtue of axial symmetry, we can express the potential as $\Phi(\mathbf{r})=\Phi(x, z) e^{i l \theta}$, where an analogous relation holds for the corresponding induced charge density. Therefore, we anticipate that the plasmon eigenmodes can be classified in terms of their angular momentum $l$, each containing a further subset of radial eigenindices $n$ that allocate the mode's type of radial confinement. Moreover, since the natural length scale of a disk is its radius, we adopt $L \equiv R$, so that we have $\Lambda=2 i \omega \varepsilon_{0} \bar{\varepsilon} R / \sigma(\omega)$ and the in-plane potential becomes $\phi\left(\tilde{\mathbf{r}}_{\|}\right)=\phi(\tilde{r}) e^{i l \theta}$, where $\tilde{r}=r / R$. Additionally, the dimensionless function $g\left(\tilde{\mathbf{r}}_{\|}, \tilde{\mathbf{r}}_{\|}^{\prime}\right)$ admits a representation of the form $g\left(\tilde{r}, \tilde{r}^{\prime}\right)=\frac{1}{2} \int_{0}^{\infty} d p J_{l}(p \tilde{r}) J_{l}\left(p \tilde{r}^{\prime}\right)$, where $J_{l}$ is the Bessel function of the first kind of order $l$. Next, we expand the radial potential inside the disk-associated with an angular momentum $l$ - using a basis in terms of Jacobi polynomials, that is, specifically, $\phi(\tilde{r})=\sum_{n=0}^{\infty} c_{n} \tilde{r}^{l} P_{n}^{(l, 0)}\left(1-2 \tilde{r}^{2}\right)$, where $P_{n}^{(l, 0)}$ denotes the Jacobi polynomials. Lastly, using the orthogonality relation of Jacobi polynomials [95], the integro-differential equation for the potential (7) can be converted into a standard eigenvalue problem as in Eq. (8). Curiously_and perhaps rather surprisingly_in the present case the matrix associated with this eigenproblem possesses analytical matrix elements [97].


Figure 4 Plasmons in an individual 2D nanodisk of radius $R$. (a) Eigenvalues for $l \geq 1$ associated with plasmon resonances of a 2D nanodisk obtained using the nonretarded framework described in the text (colored circles; the dashed lines connecting the circles are only for visual guidance). (b) Corresponding dispersion curves in the case where the 2D material's optical response is well described by a conductivity of the Drude kind [see Eqs. (9) and (10)]. Here, $\omega_{R}=\sqrt{\frac{\mathscr{D}}{2 \pi \varepsilon_{0} \bar{\varepsilon}} R^{-1}}$, where $\mathscr{D}$ is the Drude weight of the 2D electron system. (c) Spatial profiles of the normalized induced charge density for eigenmodes with different $\{l, n\}$ pairs. All results $[(\mathrm{a}-\mathrm{c})]$ were obtained by truncating the expansion to $N=100$.

The eigenvalues $\lambda_{l}^{[n]}$ characterizing the spectrum of plasmon resonances in 2D nanodisks are depicted in Figs. $4 \mathrm{a}-\mathrm{b}$ as a function of the angular momentum $l$ (where the $l=0$ modes have been omitted, and where the color is used to indicate eigenmodes with the same radial eigenindex $n$ ). Like in the ribbon configuration, the spectrum shows a manifold of eigenmodes whose energies increase with increasing eigenindex $n$; however, and contrasting the ribbon geometry, these do not form "bands", courtesy of the azimuthal quantization $l$ that has no equivalent in the ribbon structure. The nature of the disk's eigenmodes with distinct combinations of $\{l, n\}$ pairs becomes readily apparent upon inspecting 


\begin{tabular}{lccc}
\hline & $n=1$ & $n=2$ & $n=3$ \\
\hline \hline$\lambda_{l=1}^{[n]}$ & 1.0978 & 4.9141 & 8.1338 \\
\hline$\lambda_{l=2}^{[n]}$ & 1.9943 & 6.2456 & 9.5456 \\
\hline$\lambda_{l=3}^{[n]}$ & 2.8557 & 7.5125 & 10.899 \\
\hline
\end{tabular}

Table 2 Computed eigenvalues $\lambda_{l}^{[n]}$ corresponding to different $\{l, n\}$ eigenmodes that classify the plasmon resonances in individual 2D nanodisks (cf. Fig. 4).

the corresponding induced charge density profiles, as shown in Fig. 4c. The associated eigenvalues $\lambda_{l}^{[n]}$ are referenced in Table 2 for the reader's convenience. Unsurprisingly, the charge density profiles are naturally reminiscent of the displacement ascribed to the normal modes of a vibrating circular membrane, where the "quantum numbers" $l$ and $n$ classify the quantization along the azimuthal and radial directions, respectively.

The disk's eigenmodes of dipole character, i.e., with $l=1$, are of particular importance with respect to their excitation and interaction with plane-waves, since they can couple strongly to these. Evidently, in the nonretarded limit the fundamental dipole mode $\{1,1\}$ dominates the optical response of the system under plane-wave illumination, and whose absorption cross section has been shown to be quite substantial [66,98].

In passing, it should be noted that the theoretical framework outlined above does not include edges effects pertaining to the atomic termination of the 2D nanostructure's edges [79, 86, 99, 100] or quantum finite-sized effects [79] associated with extremely small nanoislands containing a few tens of atoms. In order to incorporate these effects, fully atomistic calculations (e.g., the RPA with tight-binding states or TDDFT methods) must necessarily be employed.

\subsection{Ultraconfined acoustic plasmons in hybrid graphene-dielectric-metal structures}

The remarkable degree of field confinement brought about by graphene plasmons makes them particularly well-suited for studying and enhancing light-matter interactions, and, from a practical viewpoint, can be regarded as an appealing platform for delivering miniaturized nanophotonic devices. We have seen in Sect. 2.1 that plasmons in extended graphene can attain wavelengths up to 100-300 times smaller than that of a photon of the same frequency, and that this already quite extraordinary confinement can be pushed even further by patterning graphene into plasmonic nanoresonator structures. Yet another route is to exploit the lower frequency acoustic plasmon of double-layer graphene formed by plasmon hybridization [41], since this mode reaches increasingly larger wavevectors as the separation between the graphene layers is decreased. Unfortunately, this comes hand in hand with the difficulty of exciting such graphene acoustic plasmons from the far-field owing to (i) the deep subwavelength nature of the mode itself, and (ii) to the symmetry of its field distribution (induced charges in opposite graphene layers are in anti-phase [41]). Recently, this adversity has been circumvented by using a vdW heterostructure [101] — composed of graphene encapsulated in hBN-placed onto a metal gate $[67,102,103]$. Crucially, the latter, besides enabling active control over the Fermi level of graphene, also screens the collective charge oscillations (plasmons) in the graphene monolayer that lies just above the metal, thereby yielding graphene plasmons whose properties are reminiscent of acoustic plasmons in a graphene double-layer [104].

In the following, we provide an overview of the electrodynamics governing these ultraconfined acoustic-like plasmons supported by a graphene monolayer lying at a small distance $d$ from a metal substrate. Most notably, their deeply subwavelength nature makes them highly susceptible to nonlocal effects, and thus the aforementioned modes may be used to uncover the nonlocal response of the system and to probe the quantum mechanical features of the graphene electron liquid [67] (and of the metal [104, 105]). We begin our considerations first by analyzing quantum nonlocal effects ascribed to the electrodynamics of graphene alone, and then move on towards the general case where both the graphene and the metal are treated beyond the local-response approximation.

\subsubsection{Acoustic graphene plasmons: Nonlocal effects}

The pioneering experimental works $[67,102]$ that have reported the excitation and corresponding observation of acoustic graphene plasmons (AGPs) used a setup that consisted in hBN-encapsulated graphene deposited onto an underlying metal gate (typically made of gold with a thin titanium adhesion layer). For graphene-metal separations $d$ below the penetration length of the graphene plasmon's electric field, the screening exerted by the nearby metallic gate gives rise to the above-noted AGPs, which exhibit an approximately linear plasmon dispersion at low frequencies. The spectral properties of AGPs can be measured using, for instance, a scanning near-field optical microscope (SNOM), either in scattering or in photocurrent mode [67,102].

From a theoretical standpoint, the spectral properties of AGPs can be determined by solving the associated electrodynamical problem or, alternatively, solving the quasi-static Poisson equation since the nonretarded regime is a very good approximation here. In particular, the dispersion of these screened graphene plasmons can be identified from the zeros of the RPA dielectric function [41]

$$
\varepsilon^{\mathrm{RPA}}(q, \omega)=1-V(q, \omega) \chi_{0}^{\tau}(q, \omega),
$$

where $\chi_{0}^{\tau}(q, \omega)$ is the Mermin-corrected noninteracting density-density response function of graphene [41,57] and $V(q, \omega)$ describes the dressed Coulomb interaction akin to the layered geometry.

As previously mentioned, due to the character of their dispersion relation, AGPs have the potential to achieve extremely large wavevectors. At the same time, the corresponding plasmon velocity, $v_{\mathrm{p}}$, can be tailored by controlling the 

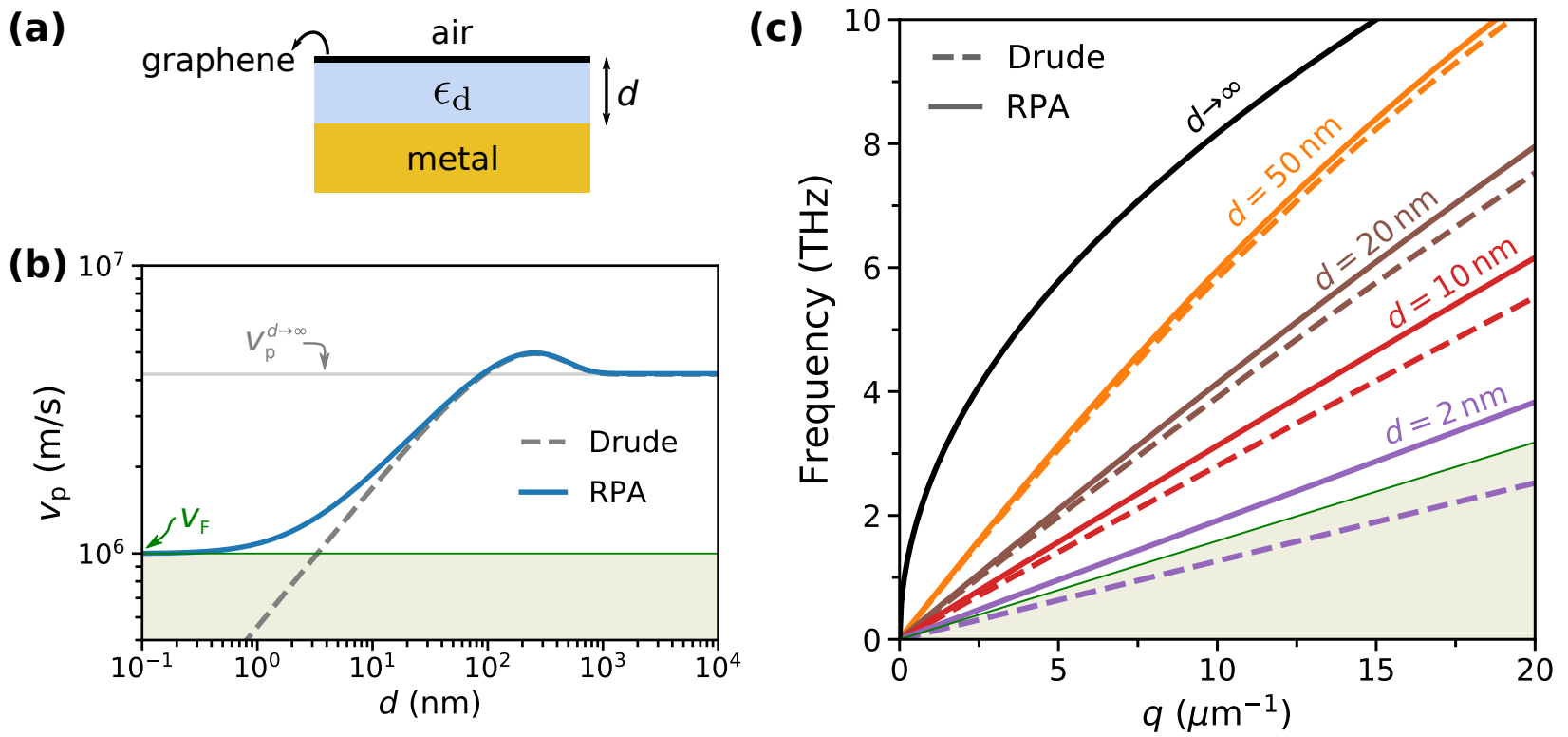

Figure 5 Transition from the local to the nonlocal response regime by controlling the thickness $d$ of the dielectric separating a graphene layer from a metal substrate. (a) Pictorial representation of the graphene-dielectric-metal heterostructure under consideration. (b) Plasmon velocity $v_{\mathrm{p}} \equiv \partial \omega / \partial q$ as a function of the graphene-metal separation $d$ (for a resonant frequency of $\omega /(2 \pi)=5 \mathrm{THz}$ ). (c) Dispersion curves of graphene plasmons supported by the graphene-dielectric-metal system for four different graphene-metal separations (indicated in the plot). The dispersion of conventional ("unscreened") graphene plasmons is shown by the solid black line to facilitate the comparison and the interpretation of the results. Setup parameters: $\varepsilon_{\mathrm{d}}=\sqrt{\varepsilon_{x}^{\mathrm{hBN}}(0) \varepsilon_{z}^{\mathrm{hBN}}(0)} \simeq 4.88[67], n_{e}=10^{12} \mathrm{~cm}^{-2}$ (which roughly amounts to $E_{F}=0.117 \mathrm{eV}$, for $v_{F}=10^{6} \mathrm{~m} \mathrm{~s}^{-1}$ ); as in Ref. [67], the metal is assumed to be well approximated by a perfect electric conductor in the $\mathrm{THz}$ regime.

distance $d$ between the graphene layer and the metallic gate. Crucially, this can be done with atomic precision since the distance $d$ amounts to the thickness of the lower hBN slab, and thus may in principle be chosen with single-atom definiteness. This feature therefore makes the graphenedielectric-metal (GDM) system considered here a unique playground to study nonlocal and quantum effects in condensed matter systems, whereby nonclassical effects can be probed and subsequently inferred from the material's plasmonic response [67].

Figure 5 explicitly shows how the dispersion of AGPs can be tailored by tuning the graphene-metal separation $d$ (the setup under consideration is portrayed in Fig. 5a). Notably, the plasmon velocity becomes increasingly slower as the graphene-metal spacing is reduced (Fig. 5b), a feature that is accompanied by a significant change of the plasmon dispersion towards larger wavevectors (Fig. 5c). Furthermore, although the classical result qualitatively captures the above-noted trends, it incurs in significant quantitative deviations from the proper nonlocal plasmon dispersion. Moreover, and perhaps more troubling, is the fact that, when the graphene-metal separation is on the order of a few nanometers, the classical theory predicts plasmon dispersion diagrams falling within the electron-hole continuum and corresponding plasmon velocities that fall below the Fermi velocity of electrons (or holes) in graphene. Clearly, such behavior is prohibited by the full nonlocal theory, the reason lying in the divergent feature of the density-density correlation function $\chi_{0}(q, \omega)$ as $q \rightarrow \omega / v_{F}$.

The calculations of the AGPs spectral properties presented in Fig. 5 underscore the importance of employing an adequate nonlocal description of plasmonic excitations in such a graphene-metal configuration, particularly when the separation between the metallic substrate and the graphene sheet enters the few-nanometer regime. In what follows, we exploit the tunability and flexibility of this heterostructureprovided by the control over the separation $d$, but also over the carrier density $n_{e}$ - to investigate the nonlocal response of the metal below the graphene sheet.

\subsubsection{Probing the nonlocal optical response of metals using graphene plasmons}

In our investigations so far we have treated the metal at the bottom of the dielectric-graphene-dielectric-metal heterostructure within the LRA. The justification for doing so has been that the dynamics affecting the graphene plasmons in the considered system are dominated by the electrodynamics of the graphene itself. Nevertheless, given the high momenta attained by the AGPs studied here, at some point the nonlocal response of the metal will need to be accounted 
for. In what follows, we consider nonlocal effects both in the electrodynamic response of graphene and the metal. In latter case, we incorporate nonlocality using the hydrodynamical model (HDM) [104, 106].

In that spirit, we consider a graphene-dielectric-metal vertical heterostructure as depicted in Fig. 6. The implicit condition for the dispersion relation of acoustic-like graphene plasmons supported by the aforementioned heterostructure, is then [104]

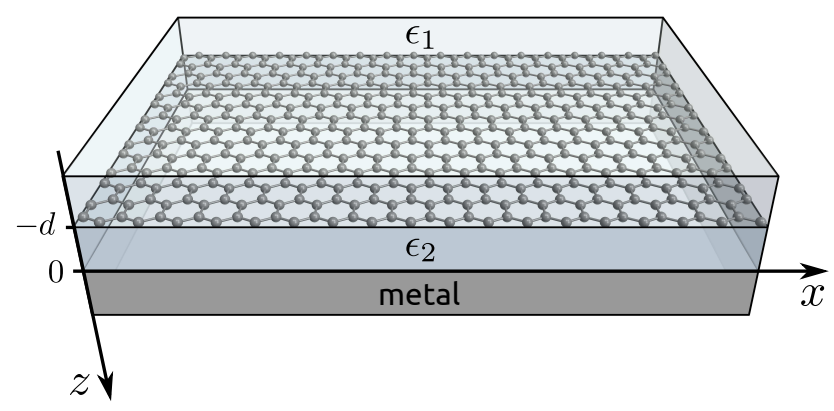

Figure 6 Graphene-dielectric-metal heterostructure. The graphene monolayer is located in the $z=-d$ plane, and it is sandwiched between two dielectric media with relative permittivities $\varepsilon_{1}$ and $\varepsilon_{2}$. The metal is assumed to exhibit a nonlocal response well modeled by the hydrodynamical model.

$$
\begin{aligned}
& \left(\frac{\varepsilon_{1}}{\kappa_{1}}+\frac{\varepsilon_{2}}{\kappa_{2}}+\frac{i \sigma}{\omega \varepsilon_{0}}\right)\left(1+\frac{\varepsilon_{\mathrm{m}} \kappa_{2}}{\varepsilon_{2} \kappa_{\mathrm{m}}}+\delta_{\mathrm{nl}}\right) \\
= & \left(\frac{\varepsilon_{1}}{\kappa_{1}}-\frac{\varepsilon_{2}}{\kappa_{2}}+\frac{i \sigma}{\omega \varepsilon_{0}}\right)\left(-1+\frac{\varepsilon_{\mathrm{m}} \kappa_{2}}{\varepsilon_{2} \kappa_{\mathrm{m}}}-\delta_{\mathrm{nl}}\right) e^{-2 \kappa_{2} d}
\end{aligned}
$$

where

$$
\delta_{\mathrm{nl}}=\frac{q^{2}}{\kappa_{\mathrm{nl}} \kappa_{\mathrm{m}}} \frac{\varepsilon_{\mathrm{m}}-\varepsilon_{\infty}}{\varepsilon_{\infty}},
$$

is a nonlocal correction. Crucially, the transcendental equation posed by the condition (12a) determines the dispersion relation of AGPs in planar dielectric-graphene-dielectricmetal structures $^{2}$ (see Fig. 6) including nonlocal effects in graphene as well as in the metal. In the former, these are accounted for via a suitable expression for the material's surface conductivity, i.e., $\sigma \equiv \sigma(q, \omega)$, whereas in the latter nonlocality enters within the scope of the HDM through the nonlocal correction parameter $\delta_{\mathrm{nl}}$.

Before proceeding further, it is instructive to take a closer look at Eq. (12). We note that the term on the left-hand side is simply the multiplication of the "bare" dispersion relations of the uncoupled interfaces: that of graphene cladded

\footnotetext{
2 If the dielectric media that encapsulate the graphene sheet are uniaxial $\stackrel{\leftrightarrow}{\varepsilon}_{j}=\operatorname{diag}\left[\varepsilon_{j}^{x}, \varepsilon_{j}^{x}, \varepsilon_{j}^{z}\right]$ (like, for instance, hBN) the corresponding AGPs' dispersion relation is nevertheless readily obtainable from Eq. (12) upon making the following replacements: $\varepsilon_{j} \rightarrow \varepsilon_{j}^{x}$ and $\kappa_{j} \rightarrow \sqrt{\left(\varepsilon_{j}^{x} / \varepsilon_{j}^{z}\right) q^{2}-\varepsilon_{j}^{x} k_{0}^{2}}$ for $j \in\{1,2\}$. This setting is considered in Ref. [104], together with the hybridization of the acoustic-like graphene plasmons with the optical phonons in hBN
}

by two dielectric media with relative permittivities $\varepsilon_{1}$ and $\varepsilon_{2}$ times that of a dielectric-metal interface. On the other hand, the term figuring on the right-hand side of Eq. (12a) mediates the interaction between the two interfaces. Clearly, in the limit of large separations between the graphene and the metal, or, more precisely, when $\kappa_{2} d \rightarrow \infty$ (corresponding to $q d \gg 1$ in the nonretarded regime), the two interfaces effectively decouple.

Figure 7 shows the AGPs' spectrum computed using three different approaches: (i) both the graphene and the metal are modeled within the LRA (this plays the role of a "base-line"), (ii) the graphene electrodynamics are described within the nonlocal RPA, whereas the metal's optical response is still treated within the LRA, and, finally, (iii) the latter assumption is relaxed further and nonlocality in the metal is taken into account under the framework of the HDM. The results unambiguously demonstrate that the incorporation of nonlocality in the metal's optical response imparts an additional blueshift to the AGPs' dispersion. In particular, the magnitude of the blueshift is proportional to the velocity of the hydrodynamic plasma pressure wave $\beta=\sqrt{3 / 5} v_{F, \mathrm{~m}}$ and inversely proportional to the metal's plasma frequency (not shown; see Ref. [104]). Hence-and provided that all other parameters are wellknown experimentally-we envision that one could use the theoretical framework outlined above in order to shed light about the metal's nonlocal response, e.g., deducing the value of $\beta$ by comparing the experimentally-measured and the theoretical spectra [103, 104, 107]. A perhaps more appropriate reasoning, that squarely rests on the microscopic formalism of the Feibelman $d$-parameters [108-110], is to establish a connection between the value of $\beta$ and the position of the centroid of the induced charge density with respect to the dielectric-metal interface (given by $\operatorname{Re} d_{\perp}$, and where $d_{\perp}$ is the main Feibelman $d$-parameter).

Next, in Fig. 8 we examine the dependence of the nonlocal hydrodynamic blueshift on the graphene-metal separation $d$. For the parameters considered in the figure (well within reach of current experimental capabilities [67, 103]), the nonlocal response of the metal can lead to quite significant blueshifts of the AGPs' dispersion for $d \lesssim 5 \mathrm{~nm}$. This can be understood by realizing that AGPs attain increasingly larger wavevectors - and therefore are more sensitive to nonlocality in general-upon decreasing $d$. Furthermore, for graphene-metal separations of only a fewnanometers, $d$ becomes comparable to the penetration length of the electric field into the metal promoted by the smearing of the electronic density and concomitant "inward spill" of the screening charges due to nonlocality [111-116]. In particular, this notion has been exploited to theoretically describe plasmonic dimers by replacing the actual dimer separation $d_{0}$ (which plays a similar role to the graphenemetal separation $d$ in our geometry) by an "effective separation" $d_{\text {eff }} \neq d_{0}$ in order to capture (albeit only qualitatively) the above-noted nonlocal shift of the induced surface charges [103, 104, 113, 114]. 
-.- G@LRA + M@LRA G@RPA + M@LRA
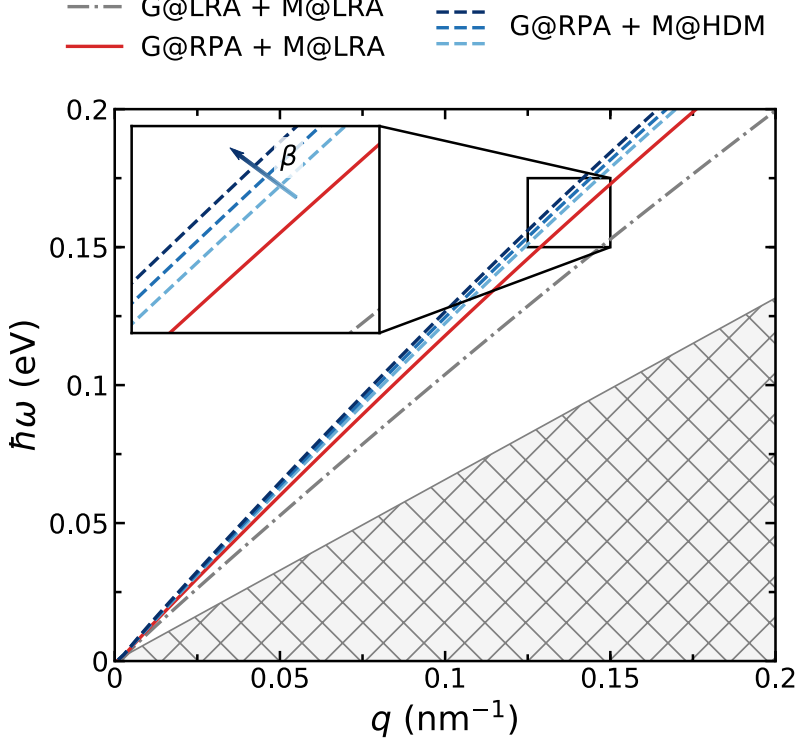

Figure 7 Nonlocal plasmonic response exhibited by acoustic-like graphene plasmons sustained at a dielectric-graphene-dielectricmetal heterostructure (cf. Fig. 6). The red solid line shows the calculation of the AGPs' spectrum in the scenario where nonlocal effects are taken into account in the optical response of graphene alone (via the nonlocal RPA with Mermin's RTA) and the metal is still treated within LRA. The blue dashed lines shows the AGPs' dispersion computed using full nonlocal electrodynamics, that is, where the optical response of the graphene and the metal are described using the nonlocal RPA and the nonlocal HDM, respectively. In the latter, we take $\beta=\sqrt{3 / 5} v_{F, \mathrm{~m}}$ with $v_{F, \mathrm{~m}}=$ $\{1,1.5,2\} \times 10^{6} \mathrm{~m} / \mathrm{s}$ (where darker shades of blue correspond to larger values of the Fermi velocity associated with the metal's conduction electrons). The opposite case, where both materials are treated within the simplest LRA is represented by the gray dot-dashed line (for the purpose of comparison). The hatched region indicates the domain associated with intraband Landau damping in the graphene. Setup parameters: $\varepsilon_{1}=1, \varepsilon_{2}=4$, and $d=2 \mathrm{~nm}$; the graphene is modeled with the parameters $E_{F}=$ $0.4 \mathrm{eV}$ and $\hbar \gamma=8 \mathrm{meV}$; the metal is modeled in accordance with $\varepsilon_{\mathrm{m}}=1-\omega_{\mathrm{p}}^{2} /\left(\omega^{2}+i \omega \gamma_{\mathrm{m}}\right)$ with $\hbar \omega_{\mathrm{p}}=9 \mathrm{eV}, \hbar \gamma_{\mathrm{m}}=20 \mathrm{meV}$ (and $\beta=\sqrt{3 / 5} v_{F, \mathrm{~m}}$ for the HDM).

\section{Nonlinear graphene plasmonics}

Nonlinear optical phenomena enable the temporal and spectral diversification of laser light required in a plethora of modern photonic functionalities, yet are limited by the inherently weak nonlinear response offered by conventional materials $[117,118]$. While this situation can be remedied in bulk crystals by propagating light over a distance of many optical wavelengths while meeting stringent phase-matching conditions, the same strategy cannot be applied to nanophotonic structures with inherently small light-matter interaction volumes. For this reason, the ability of polaritons to concentrate light on deep subwavelength scales, intensifying the electromagnetic near-fields upon which the nonlinear optical re-

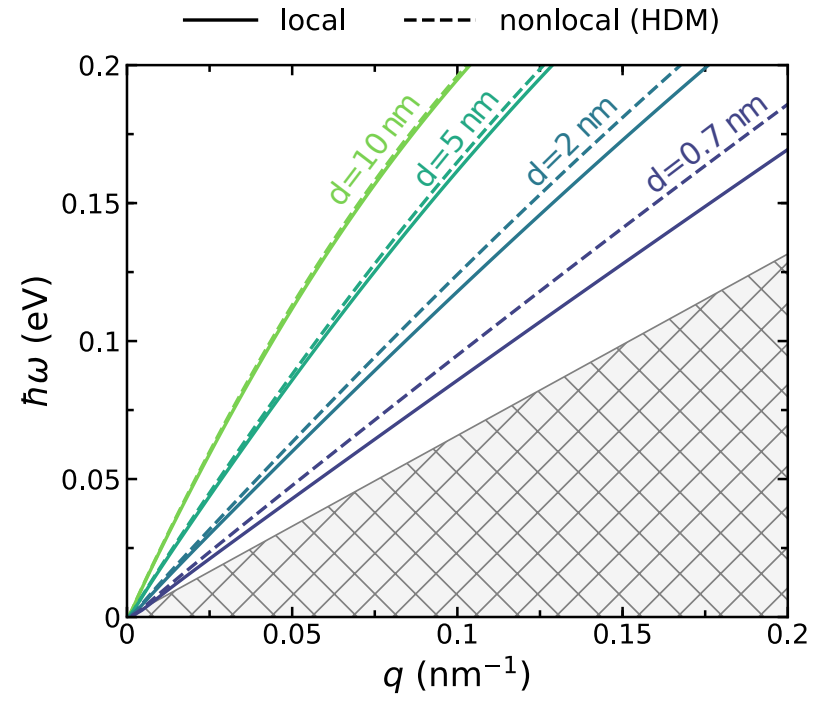

Figure 8 Dependence of the nonlocal plasmonic response of acoustic-like graphene plasmons for varying graphene-metal separations $d$ (indicated in the plot with matching colors). The response of graphene is evaluated at the level of the nonlocal RPA in all cases shown here. As for the metal's optical response, we consider two distinct models: the local response approximation (solid lines) and nonlocal hydrodynamic model (dashed lines). Setup parameters: same as in Fig. 7 but with fixed $\beta=\sqrt{3 / 5} v_{F, \mathrm{~m}}$, where $v_{F, \mathrm{~m}}=1.4 \times 10^{6} \mathrm{~m} \mathrm{~s}^{-1}$ (as in gold or silver).

sponse superlinearly depends, garners considerable attention in nonlinear nano-optics. In this context, the extreme electric field enhancement provided by plasmon polaritons in noble metal nanostructures motivates intensive research efforts in the field of nonlinear plasmonics [119], with plasmon resonances providing substantial enhancements in the effective nonlinear optical response associated with all-optical modulation [120,121], wave-mixing [122-124], and harmonic generation $[125,126]$, thereby facilitating the control of light by light on the nanoscale.

The strong intrinsic field enhancement provided by the long-lived and electrically-tunable plasmons of monolayer graphene (cf. Sect. 2) has naturally launched explorations of its ability to enhance nonlinear optical phenomena, stimulating fertile research efforts in nonlinear graphene plasmonics [85, 127-149]. Fortuitously, the unique linear electronic dispersion relation of graphene, giving rise to its universal $2.3 \%$ broadband light absorption and facile electrical tunability, also endows this 2D material with an intrinsically anharmonic response to external electromagnetic fields: lowenergy charge carriers within a single Dirac cone have energies $\varepsilon_{\mathbf{k}}=\hbar v_{\mathrm{F}}|\mathbf{k}|$, where $\mathbf{k}$ is the electron wavevector and $v_{\mathrm{F}} \approx c / 300$ is the Fermi velocity, endowing them with a velocity of fixed magnitude that instantaneously changes sign when crossing the Dirac point; an applied ac electric field $E(t)=E_{0} \cos (\omega t)$ thus leads to a square-wave surface current density $J(t)=-e n v_{\mathrm{F}} \operatorname{sign}\{\sin (\omega t)\}$ in the $E_{0} \rightarrow \infty$ limit that is weighted by the charge carrier density 
(a)
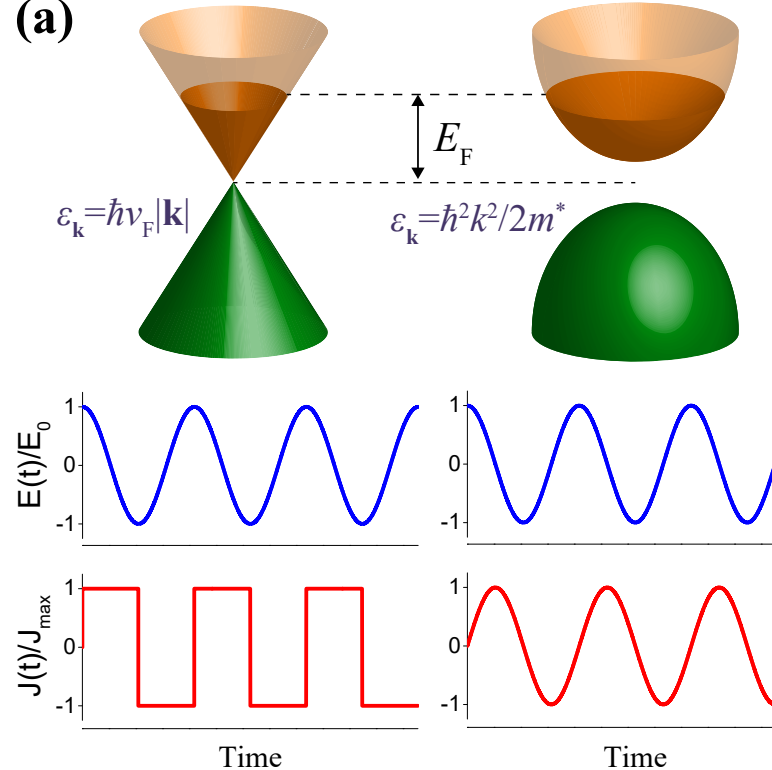

Time
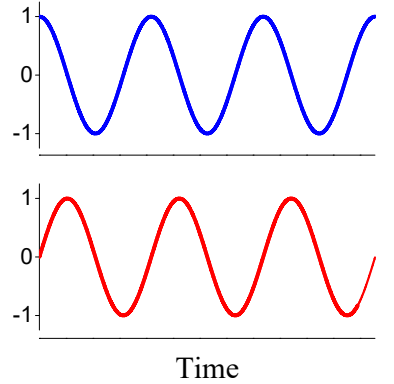

(b)



Figure 9 Nonlinear graphene optics and plasmon-assisted high-harmonic generation. (a) The conical low-energy band structure of graphene (upper left) responds to a monochromatic electric field $E(t)=E_{0} \cos (\omega t)$ by producing an induced current with a square-wave temporal profile in the $E_{0} \rightarrow \infty$ limit, containing all odd-ordered harmonics in its Fourier spectrum; in contrast, a semiconducting crystal with parabolic charge carrier dispersion (upper right) responds to the same field harmonically at the driving frequency $\omega$. (b) The contour plot shows the simulated light emission (dipole acceleration) from a $20 \mathrm{~nm}$-wide graphene nanoribbon doped to $E_{\mathrm{F}}=0.4 \mathrm{eV}$ when driven by a transverse-polarized normally-incident light pulse as a function of the incident and emitted photon energies. The impinging pulse is characterized by a peak intensity of $10^{12} \mathrm{~W} / \mathrm{m}^{2}$ and full width at half maximum (FWHM) duration of $100 \mathrm{fs}$. In the adjacent panels the absorption cross-section normalized to the ribbon area is indicated along both axes of the contour plot by filled orange curves, with the emission produced upon resonant illumination of the nanoribbon plasmon plotted as a purple curve in the right panel, where the pulse is centered spectrally at $\hbar \omega_{\mathrm{p}}=0.336 \mathrm{eV}$ (i.e., at the energy indicated by the vertical dashed line in the contour). Adapted with permission. [127] Copyright 2017, Springer Nature.

$n$ and contains significant contributions from all odd harmonics in its Fourier decomposition (see Fig. 9a) [150, 151]. However, despite a large optical nonlinearity associated with intraband charge carrier motion near the Dirac point, most experiments in graphene nonlinear optics have only probed the interband response linking vertical transitions between Dirac cones [152-160], presumably due to the availability of interband transitions at all photon energies that could potentially enhance nonlinear processes involving multiple light frequencies, and the wider abundance of high-powered lasers operating in the near-IR and visible regimes that are routinely employed in nonlinear optical experiments. The initial report of a large optical nonlinearity in graphene was demonstrated in a fourwave-mixing experiment using intense near-IR/visible light pulses [152], followed by explorations of harmonic generation [153, 156-158], the optical Kerr effect [154], and other four-wave-mixing schemes [155], with reported values of the third-order nonlinear susceptibility $\chi^{(3)}$ ranging from $\sim 10^{-15}-10^{-19} \mathrm{~m}^{2} / \mathrm{V}^{2}\left(\sim 10^{-7}-10^{-11}\right.$ esu in Gaussian units) [161]. The ability to electrically modulate the nonlinear optical response in graphene has recently been demonstrated [159, 160], along with high-harmonic generation by the carbon monolayer when driven by intense terahertz [162] and mid-IR [163, 164] light pulses.
Early theoretical ventures in nonlinear graphene plasmonics employed perturbation theory to reveal large secondharmonic [128] and Kerr-type optical nonlinearities [129] associated with the highly-confined surface plasmon polaritons hosted by extended graphene, which however cannot be directly launched by light from free space due to their large momenta. Nonlinear optical wave-mixing was later predicted to circumvent this limitation and enable the excitation of graphene plasmons with visible light impinging at angles such that a linear combinations of their frequency and in-plane optical momentum coincides with the plasmon dispersion curve lying well-below the light line [131,132]. Further strengthening light-matter coupling to boost the intrinsically-large graphene nonlinearity, localized plasmons in graphene nanoresonators, concentrating light to volumes millions of times smaller than its corresponding free-space wavelength, are predicted to enable single-photon-level nonlinear optical interactions [130, 136, 147], optical bistability [135], and intense nonlinear absorption [134,145]. These exciting prospects were explored in classical electrodynamic models of the nonlinear optical response associated with plasmons in graphene nanostructures, characterizing the optical response using the linear and nonlinear optical conductivities of extended graphene; In parallel, atomistic simulations of plasmons in nanostructured graphene, with the 
dynamics of electrons in tight-binding (TB) states governed by a single-particle density matrix in the self-consistent field approximation — at the level of the RPA — reveal enormous nonlinear polarizabilities associated with harmonic generation [85] and wave-mixing [133] processes (orders of magnitude above those produced by plasmons in similarlysized noble metal nanoparticles), confirming that the excellent nonlinear optical properties of graphene persist in its nanostructured form, and identifying the thresholds at which quantum finite-size effects emerge in structures with lateral size below $\sim 10 \mathrm{~nm}$ [139]. Time-domain simulations based on the TB+RPA method, which go beyond perturbation theory to enable the exploration of extreme nonlinear optical processes driven by intense ultrashort optical pulses, present further evidence of plasmonic enhancement in high-harmonic generation produced by doped graphene nanoribbons [127], as we show in Fig. 9(b). We refer the interested reader to Ref. [149] for a review on the nonlinear TB+RPA approach in nanostructured graphene. For larger 2D nanostructures, the nonlinear optical response can be well-described by the theoretical description outlined in the following subsection, designed to model localized graphene plasmons but applicable to polaritons in other atomically-thin materials characterized by 2D linear and nonlinear optical conductivities. Going beyond perturbation theory, we later discuss the regime of extreme nonlinear plasmonics, where intense optical pulses resonantly drive plasmon-enhanced saturable absorption and high-harmonic generation.

\subsection{Analytical description of plasmon-assisted nonlinearity in graphene nanostructures}

The nonlinear plasmonic response in graphene nanostructures of $\gtrsim 10 \mathrm{~nm}$ lateral size, i.e., beyond the threshold at which quantum finite-size effects play a non-negligible role [79], can be described semi-analytically through the procedure introduced in Ref. [141], which constitutes an extension of the electrostatic eigenmode expansion for the linear optical response outlined in Ref. [66] (in a way reminiscent of the framework detailed in Sect. 2.2.1) to higher-order processes. In this model (presented in this section in Gaussian unit) the response associated with a nonlinear optical process of order $n$ and harmonic $s$ (e.g., $n=s=3$ for third harmonic generation) to an external field $\mathbf{E}^{\mathrm{ext}} \mathrm{e}^{-\mathrm{i} \omega t}+$ c.c. polarized in the $\mathbf{r}_{\|}=(x, y)$ plane of the graphene nanostructure, with geometry defined by the filling function $f\left(\mathbf{r}_{\|}\right)$, is characterized by the induced dipole moment

$$
\mathbf{p}^{n, s}=\frac{\mathrm{i}}{s \omega} \int d \mathbf{r}_{\|} \mathbf{j}^{n, s}\left(\mathbf{r}_{\|}\right),
$$

where the total current

$$
\mathbf{j}^{n, s}=\mathbf{j}_{\mathrm{NL}}^{n, s}+\sigma_{s \omega}^{(1)} \mathbf{E}^{n, s},
$$

contains contributions from the intrinsic nonlinear current $\mathbf{j}_{\mathrm{NL}}^{n, s}$ driven by the linear electric field

$$
\mathbf{E}^{1,1}\left(\mathbf{r}_{\|}\right)=\mathbf{E}^{\mathrm{ext}}+\frac{\mathrm{i} \sigma_{\omega}^{(1)}}{\omega \bar{\varepsilon}} \nabla_{\|} \int \frac{d \mathbf{r}_{\|}^{\prime}}{\left|\mathbf{r}_{\|}-\mathbf{r}_{\|}^{\prime}\right|} \nabla_{\|}^{\prime} \cdot f\left(\mathbf{r}_{\|}^{\prime}\right) \mathbf{E}^{1,1}\left(\mathbf{r}_{\|}^{\prime}\right)
$$

at the impinging light frequency $\omega$ and the current arising from the system's linear response to the self-consistent nonlinear field

$$
\mathbf{E}^{n, s}\left(\mathbf{r}_{\|}\right)=\frac{\mathrm{i}}{s \omega} \boldsymbol{\nabla}_{\|} \int \frac{d \mathbf{r}_{\|}^{\prime}}{\left|\mathbf{r}_{\|}-\mathbf{r}_{\|}^{\prime}\right|} \nabla_{\|}^{\prime} \cdot \mathbf{j}^{n, s}\left(\mathbf{r}_{\|}^{\prime}\right)
$$

at the generated frequency $s \omega$, while the form of the intrinsic nonlinear current $\mathbf{j}_{\mathrm{NL}}^{n, s}$ depends on the particular nonlinear process under consideration. For an inversion-symmetric graphene nanostructure, third-order processes constitute the leading contribution to the nonlinear response due to the inherent centrosymmetry of graphene's hexagonal lattice; here we limit our discussion to third-harmonic generation (THG) and the optical Kerr nonlinearity, for which the associated currents are written as

$$
\begin{aligned}
& \mathbf{j}_{\mathrm{NL}}^{3,3}=\sigma_{3 \omega}^{(3)} \mathbf{E}^{1,1}\left(\mathbf{E}^{1,1} \cdot \mathbf{E}^{1,1}\right), \\
& \mathbf{j}_{\mathrm{NL}}^{3,1}=\sigma_{\omega}^{(3)}\left[\mathbf{E}^{1,-1}\left(\mathbf{E}^{1,1} \cdot \mathbf{E}^{1,1}\right)+2 \mathbf{E}^{1,1}\left|\mathbf{E}^{1,1}\right|^{2}\right],
\end{aligned}
$$

in terms of the local 2D nonlinear conductivities $\sigma_{s \omega}^{(n)}$ calculated for extended graphene, obtained in the single-band approximation by solving the semi-classical Boltzmann transport equation $[141,150,165]$ or in quantum-mechanical approaches that incorporate effects both due to intraband and interband electron motion [161, 166, 167].

For a given 2D morphology and specific nonlinear optical process, the self-consistent solution of Eq. (16) through the eigenmode expansion procedure detailed in Ref. [141] yields the nonlinear current $\mathbf{j}^{n, s}$ entering the expression for the induced dipole in Eq. (13). The analysis is simplified considerably by retaining only the dominant lowest-order dipolar plasmon mode in the expansion, choosing an inversionsymmetric geometry that suppresses any even-ordered nonlinear optical response, and considering normally-impinging light; under these conditions, the non-vanishing linear and nonlinear polarizabilities for a structure of characteristic size $D$ are then given by

$$
\begin{aligned}
& \alpha_{\omega}^{(1)}=\frac{\mathrm{i} D^{2}}{\omega} \sigma_{\omega}^{(1)} \frac{\zeta^{2}}{1-\eta(\omega) / \eta_{1}} \\
& \alpha_{3 \omega}^{(3)}=\frac{\mathrm{i} D^{2}}{3 \omega} \sigma_{3 \omega}^{(3)} \frac{\xi^{3,3}}{\left[1-\eta(\omega) / \eta_{1}\right]^{3}}, \\
& \alpha_{\omega}^{(3)}=\frac{\mathrm{i} D^{2}}{\omega} \sigma_{\omega}^{(3)} \frac{\xi^{3,1}}{\left[1-\eta(\omega) / \eta_{1}\right]^{2}\left|1-\eta(\omega) / \eta_{1}\right|^{2}}
\end{aligned}
$$

where $\eta(\omega)=\mathrm{i} \sigma_{\omega}^{(1)} / \bar{\varepsilon} \omega D$ contains the dependence on the linear conductivity $\sigma_{\omega}^{(1)}$ and size, while the eigenvalue $\eta_{1}$, the linear dipole coupling parameter $\zeta$, and the nonlinear 
coupling parameters $\xi^{n, s}$ are specific to the $2 \mathrm{D}$ geometry under consideration, and have been tabulated for various morphologies (e.g., disk, triangle, nanoribbon) in Ref. [141].

While the above formalism is graphene-centered, it is important to recognize that the procedure is general to any 2D material characterized by isotropic, local conductivities. In the case of graphene, the third-order conductivities associated with purely intraband charge carrier motion can be readily obtained from a perturbative solution of the Boltzmann transport equation in the local approximation as [141]

$$
\begin{aligned}
\sigma_{3 \omega}^{(3)} & =\frac{3 \mathrm{i} e^{4} v_{\mathrm{F}}^{2}}{4 \pi \hbar^{2} E_{\mathrm{F}}} \frac{1}{\left(\omega+\mathrm{i} \tau^{-1}\right)\left(2 \omega+\mathrm{i} \tau^{-1}\right)\left(3 \omega+\mathrm{i} \tau^{-1}\right)} \\
\sigma_{\omega}^{(3)} & =\frac{-3 \mathrm{i} e^{4} v_{\mathrm{F}}^{2}}{4 \pi \hbar E_{\mathrm{F}}} \frac{1}{\left(2 \omega+\mathrm{i} \tau^{-1}\right)\left(\omega^{2}+\tau^{-2}\right)}
\end{aligned}
$$

Analytical corrections to the above conductivities including interband contributions have been reported by Mikhailov [167], and constitute an extension of the local RPA description of graphene to third-order.

In Fig. 10 we employ the above procedure, incorporating the third-order local RPA conductivities reported in Ref. [167], to study plasmon-assisted third-order nonlinear optical processes in graphene nanoribbons characterized by a width $D=40 \mathrm{~nm}$ and $E_{\mathrm{F}}=1.0 \mathrm{eV}$ doping, contrasting results of the analytical model (dashed curves) with those predicted in rigorous atomistic simulations of the linear and nonlinear polarizabilities (solid curves). The four parameters $\eta_{1}=-0.06873, \zeta=0.9428, \xi^{3,3}=-0.9415$, and $\xi^{3,1}=3.093$ associated with the lowest order $(j=1)$ dipolar plasmon mode of the nanoribbon geometry already yield excellent agreement with the TB+RPA method, and can be improved by including higher-order modes.

\subsection{Extreme nonlinear plasmonics}

The strong electromagnetic field concentration provided by 2D plasmons in graphene reduces the light-intensity threshold for extreme nonlinear optical phenomena, arising when strong optical fields produce highly out-of-equilibrium electron dynamics that cannot be understood within in a pertubative theoretical framework. High harmonic generation (HHG) is one such process, predicted to be particularly effective in highly-doped, nanostructured graphene due to the synergetic combination of plasmonic near-field enhancement and anharmonic intraband charge carrier motion near the Dirac point [127] (see Fig. 9b). Plasmon-assisted HHG in graphene is however competing with its strong intrinsic saturable absorption [168], which diminishes the in-plane plasmonic near-field enhancement in the carbon layer; the coherent saturable absorption is augmented by an incoherent saturation originating in the optical heating of electrons, which becomes substantial due to enhanced light absorption at the plasmon resonance [143].

Several experimental investigations probing nonlinear plasmonic phenomena in both extended and graphene and structured resonators with intense pulsed laser fields are highlighted in Fig. 11. In particular, the electronic heating associated with resonant excitation of plasmons constitutes an incoherent but substantial source of optical nonlinearity that can be exploited to realize all-optical switching with relatively low-fluence pulses. This concept has been studied experimentally for propagating plasmons in extended graphene [169] and localized terahertz plasmons in ribbons [138]. Intense optical nonlinearities associated with wave-mixing have also been observed in experiments, originally in extended graphene as a means to excite propagating plasmons directly from free space [137], and later for nanoribbons in the near- and mid-infrared [142]; these studies also report a large effect attributed to the electronic heating produced by intense illumination.

The appeal of highly-confined and actively-tunable plasmons in graphene is partially offset by their limitation to mid-IR and lower frequencies, which presents a technological challenge impeding its widespread application towards nonlinear nano-optical devices. With plasmon resonance frequencies in graphene nanostructures scaling as $\omega \propto \sqrt{E_{\mathrm{F}} / D}$ and practical considerations limiting the graphene doping level to $E_{\mathrm{F}} \sim 1 \mathrm{eV}$, one possibility to boost plasmon resonances to near-IR and visible frequencies consists in patterning graphene on very small length scales, down to the molecular plasmonics regime, where graphene nanoislands are more akin to polycyclic aromatic hydrocarbons [170]. Interestingly, TB+RPA simulations for such graphene nanoislands down to sizes of only a few nanometers (in agreement with more sophisticated quantum chemistry simulations based on time-dependent density-functional theory) indicate that a large plasmon-assisted optical nonlinearity is maintained despite a breakdown of the Dirac cone electronic dispersion relation into discrete states separated by large energy gaps [149], suggesting molecular self-assembly [171, 172] as a path towards mid-IR nonlinear plasmonic devices. Graphene plasmons however constitute only one element within the broader suite of $2 \mathrm{D}$ polaritons that can undergo strong nonlinear optical interactions: As we discuss in the following section, exciton polaritons in TMDCs exhibit resonance energies determined by the electronic band gap of the material and electron-hole interaction, and their strong coupling with proximal external plasmonic elements leads to the formation of hybridized exciton polaritons that have their own intrinsic optical nonlinearity.

\section{Strong light-matter interactions in layered transition metal dichalcogenides}

The manipulation of light and matter is a foundational theme in photonics and plasmonics that enables their applications in integrated light sources (conventional and quantum) [173], information processing [173], as well as sensing and metrology [174]. The traditional approach is to utilize a photonic cavity to modify the electromagnetic environment of quantum light emitters such as natural atoms [175] or artificial emitters such as quantum dots based on semiconducting materials [173]. Depending on the strength of the cou- 



Figure 10 Linear and nonlinear optical response of graphene nanoribbons. For a highly-doped $\left(E_{\mathrm{F}}=1 \mathrm{eV}\right)$ graphene nanoribbon of width $D=40 \mathrm{~nm}$ we present (a) the linear absorption cross-section $\sigma^{\text {abs }}=4 \pi \omega \operatorname{Im}\left\{\alpha_{\omega}^{(1)}\right\} / c$ along with the nonlinear susceptibilities $\chi_{s \omega}^{(n)}=\alpha_{s \omega}^{(n)} / d_{\mathrm{gr}} D$ (normalizing the polarizability per unit length by the ribbon width and graphene effective thickness $d_{\mathrm{gr}}=0.33 \mathrm{~nm}$ ) for (b) third-harmonic generation ( $n=s=3$ ) and (c) the optical Kerr nonlinearity $(n=3, s=1)$, comparing atomistic quantum-mechanical (QM) simulations for zigzag-edge-terminated ribbons (solid curves) with the classical (CL) analytical theory (broken curves); in the latter, dashed curves are obtained by limiting the modal expansion to the leading-order dipolar mode, while dash-dotted curves show the response including up to the first three dipole-active modes. Reproduced with permission. [141] Copyright 2018, American Physical Society.

(a)



(c)

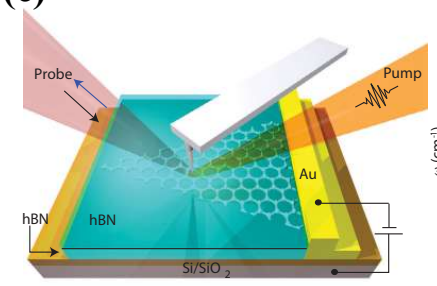

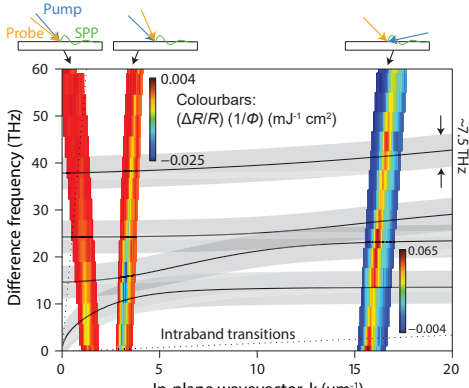

In-plane wavevector, $k\left(\mu \mathrm{m}^{-1}\right)$

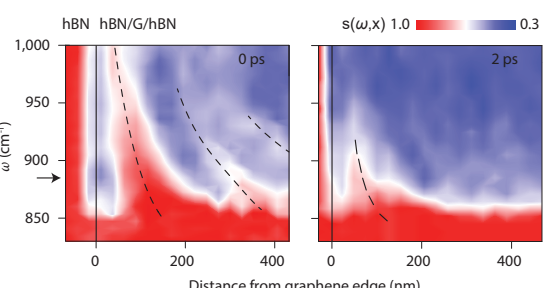

(b)
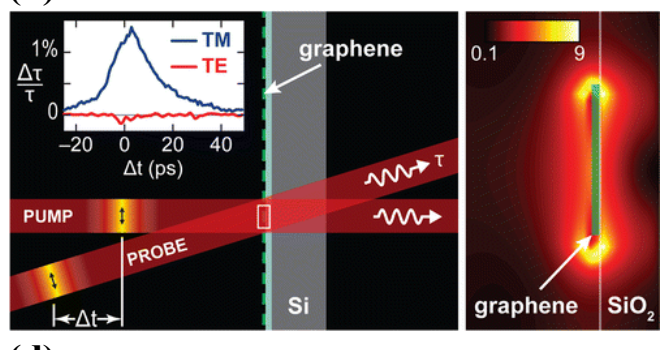

(d)

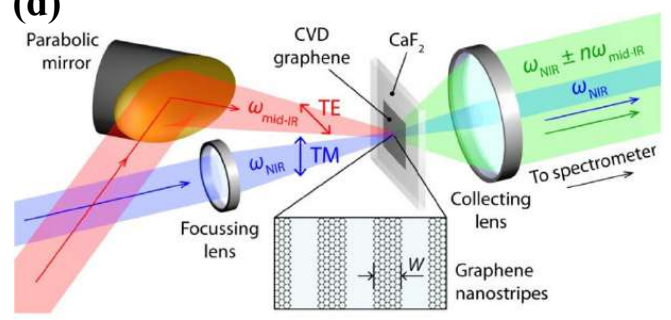

Figure 11 Experimental investigations in nonlinear graphene plasmonics. (a) The left panel illustrates conceptually the prospect of optically exciting propagating plasmons in extended graphene through nonlinear wave-mixing (difference frequency generation), which can provide the momentum required to access the graphene plasmon dispersion curve lying well-outside the light line (red curve); In the right panel the experimental signature of this process is observed through differential reflection as a function of the temporal overlap between pump and probe pulses. Adapted with permission. [137] Copyright 2016, Springer Nature. (b) Pump-probe excitation of $\mathrm{THz}$ graphene plasmons in microribbon arrays reveals a strong transient nonlinear optical response associated with electronic heating. Reproduced with permission. [138] Copyright 2016, American Chemical Society. (c) Infrared nano-imaging of hBN-encapsulated graphene reveals transient plasmons produced by femtosecond optical pump pulses, enabling real-space snapshots of the out-of-equilibrium electron dynamics. Adapted with permission. [169] Copyright 2016, Springer Nature. (d) Experimental scheme to study plasmon-assisted nonlinear wave-mixing in graphene nanoribbons. Reproduced with permission. [142] Copyright 2018, American Chemical Society.

pling between the two subsystems we can distinguish two regimes, namely the weak and the strong-coupling regimes as explained in Sect. 4.1 and illustrated in Fig. 12. In the for- mer regime, the rate of energy exchange between the emitter and the cavity is slower than any losses pertaining to the system's constituents, and thus the light and matter states 
(a)

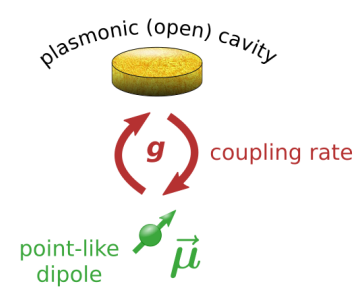

(b)

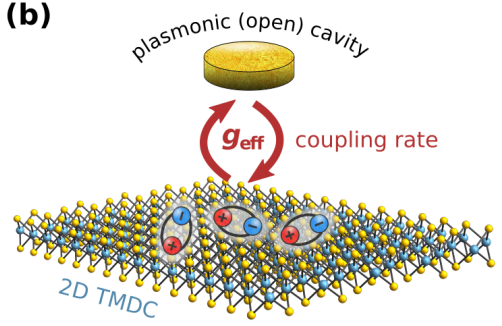

(c)
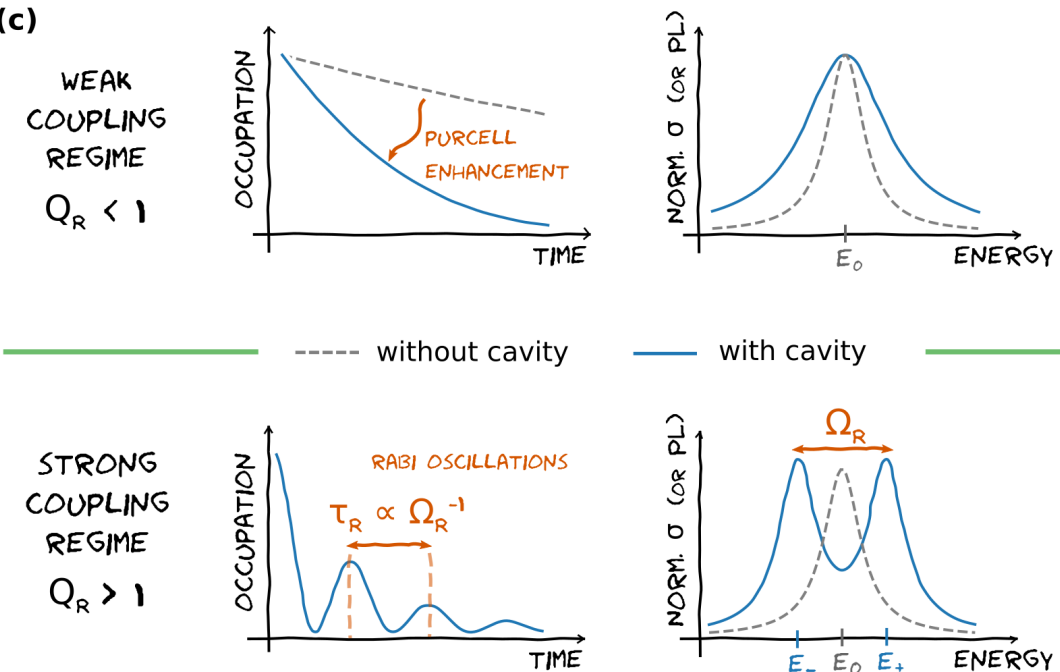

with cavity



Figure 12 Fundamentals of strong coupling in nanophotonics. (a) Plasmonic open cavity, e.g., a plasmonic resonator of some arbitrary shape (here exemplified by a gold nanodisk for definiteness alone), characterized by a plasmon resonance of energy $E_{\mathrm{pl}}$ and damping $\Gamma_{\mathrm{pl}}$, placed in the vicinity of a point-like emitter (e.g., a canonical two-level system) characterized by a transition energy $E_{\text {ex }}$, intrinsic linewidth $\Gamma_{\mathrm{ex}}$, and dipole moment $\boldsymbol{\mu}$. Here the coherent exchange of energy between the two systems is described by the coupling strength $g$. (b) Same plasmonic open cavity as in (a) but now near an atomically-thin TMDC; the latter supports excitonic resonances that play the role previously assigned to the two-level system. The coupling between the two entities is now described by an effective coupling rate $g_{\text {eff }}$ (that parameterizes the overall coupling due to an effective ensemble of excitons hosted by the 2D TMDC). (c) Pictorial representation of the interaction-modified dynamics of the emitter (here a two-level system for simplicity). In the so-called weak coupling regime, defined by $Q_{\mathrm{R}}<1$ (see text), the emitter decay rate is enhanced (Purcell enhancement) without a substantial modification of the emitter (eigen)state. Conversely, in the strong-coupling regime, i.e., for $Q_{\mathrm{R}}>1$, the coupling is so large that it leads to the formation of two new eigenstates exhibiting hybrid emitter-cavity character(s), with energy being exchanged coherently at a rate governed by the Rabi frequency $\Omega_{\mathrm{R}}$ (which amounts to $\hbar \Omega_{\mathrm{R}} \approx 2 g$ in the low-loss regime).

essentially remain as separate entities (albeit with modified dynamics). In the weak coupling regime, the enhancement of the electromagnetic local density of states (LDOS) and of the field enhancement provided by the optical cavity can be used to increase the emission rate of an emitter: this behavior is known as the Purcell effect [176-179]. On the opposite end, that is, in the strong-coupling regime-where the rate of energy exchange between the emitter and the cavity is faster than any losses - the light and matter states hybridize to form new eigenstates simultaneously endowed with both light-like and matter-like character [180]. The hybrid nature of polaritons emerging in the strong-coupling regime offers many exciting opportunities not only for fundamental research [181] but also for quantum technologies such as photon blockades [182], coherent quantum bit manipulation [183], or new quantum light generation and thresholdless polaritonic lasers [184]. However, in order to prevent detrimental dephasing processes in both the cavity and in the emitter, pioneering experimental works [185-187] have been carried out under cryogenic conditions [186,187], thus limiting the development of scalable, application-oriented devices.

Over the last few years, there has been a growing interest in studying the strong-coupling regime using organic semiconductors [187-190] and, more recently, atomically- thin and two-dimensional materials [34, 191-200] as material platforms. Here, we give special emphasis to the latter. In particular, 2D materials like TMDCs can host excitons-electron-hole pairs bounded by the (screened) Coulomb interaction-that are stable at room temperature, thereby opening enticing prospects for exploring strong light-matter interactions under ambient conditions. Strong coupling at room temperature using dielectric cavities together with TMDCs [34] has been experimentally demonstrated, while similar systems such as metallic microcavities coupled to TMDCs [201] have also joined the race, exhibiting polaritonic splittings as large as $100 \mathrm{meV}$ [202]. Futhermore, Rabi-like splittings in excess of $100 \mathrm{meV}$ have been achieved in systems utilizing plasmonic lattices and localized surface plasmons resonances (LSPRs) together with single inorganic quantum dots [203-206] or organic molecules [207] such as J-aggregates [208-211], and 2D materials [193-200]. At the time of writing, most of the reviews on strong coupling in nanophotonics [195,212-215] predominately cover previous works where the emitters are either conventional quantum dots or (in)organic molecules. Hence, herein we focus on reviewing the recent developments concerning strong light-matter interactions based on plasmonic resonances jointly with atomically-thin materials, like TMDCs, as a growing number of experimental 
works have demonstrated sizable Rabi-like energy splittings [193-200,216] (e.g., as large as $175 \mathrm{meV}$ [199] and $190 \mathrm{meV}$ [217]) using architectures based on few-layer and single-layer TMDCs. In the following, we provide an overview of the elementary principles describing strong coupling in a nanophotonics context. Next, we describe basic optical properties of TMDCs and then review the most recent experimental investigations of strong coupling between excitons in atomically-thin TMDCs and plasmonic resonances supported by metallic nanoparticles. In closing, we identify and discuss potential avenues for future investigations in this emerging and enticing field.

\subsection{The strong-coupling regime}

Let us consider a plasmonic resonator-e.g., an individual metallic nanoparticle supporting a LSPR - in close proximity to an emitter such that there is significant overlap between the near-fields of the plasmonic cavity and of the emitter. If the emitter is well described by a point-like twolevel system (Fig. 12a), then the coupling energy follows from $g=\boldsymbol{\mu} \cdot \mathbf{E}\left(\mathbf{r}_{0}\right)$, where $\boldsymbol{\mu}$ is transition dipole moment associated with the transition and $\mathbf{E}\left(\mathbf{r}_{0}\right)$ is the electric field evaluated at the emitter's position $\mathbf{r}_{0}[195,212,213,215]$. In this review, we shall consider that the "emitter" is represented by an excitonic resonance ascribed to the 2D TMDC (Fig. 12b); in such a case, the previous expression cannot be employed and an explicit formula for the coupling energy is in general a nontrivial and onerous task (for a discussion about this predicament, see, for instance, Ref. [218] and references therein). Notwithstanding, one may still in principle define an effective coupling ( $g \equiv g_{\text {eff }}$ herein) that could be inferred from experimental data.

In what follows, we treat the plasmon-exciton interaction semiclassically within the framework of the coupledoscillator model [34, 192, 215], wherein the coupling between the plasmon and the exciton is described by the nonHermitian Hamiltonian

$$
\left[\begin{array}{cc}
E_{\mathrm{pl}}-\mathrm{i} \frac{\Gamma_{\mathrm{pl}}}{2} & g \\
g & E_{\mathrm{ex}}-\mathrm{i} \frac{\Gamma_{\mathrm{ex}}}{2}
\end{array}\right]\left[\begin{array}{l}
\alpha \\
\beta
\end{array}\right]_{ \pm}=E_{ \pm}\left[\begin{array}{l}
\alpha \\
\beta
\end{array}\right]_{ \pm},
$$

where $E_{\mathrm{pl}(\mathrm{ex})}$ and $\Gamma_{\mathrm{pl}(\mathrm{ex})}$ are, respectively, the plasmon (exciton) resonant energy and associated linewidth, $g$ denotes the coupling strength introduced above, and we introduce the amplitudes $\alpha$ and $\beta$ describing the hybrid polaritonic states in terms of their plasmonic $\left(\alpha_{ \pm}\right)$and excitonic $\left(\beta_{ \pm}\right)$ constituents. The system's eigenmodes can be straighforwardly calculated by diagonalizing the Hamiltonian (24), which yields the following pair of eigenenergies:

$$
\begin{aligned}
E_{ \pm}=\frac{1}{2}( & \left.E_{\mathrm{pl}}+E_{\mathrm{ex}}\right)-\frac{\mathrm{i}}{4}\left(\Gamma_{\mathrm{pl}}+\Gamma_{\mathrm{ex}}\right) \\
& \pm \frac{1}{2} \sqrt{4 g^{2}+\left[\delta-\frac{i}{2}\left(\Gamma_{\mathrm{pl}}-\Gamma_{\mathrm{ex}}\right)\right]^{2}}
\end{aligned}
$$

where $\delta=E_{\mathrm{pl}}-E_{\mathrm{ex}}$ is the plasmon-exciton detuning. The eigenvalues $E_{ \pm}$give rise to an energy spectrum divided into two branches: an upper-polariton branch (UPB; $\left.E_{+}\right)$and a lower-polariton branch (LPB; $E_{-}$). The Rabi-like splitting, $E_{\mathrm{R}}$, is then defined as the energy difference between the UPB and LPB at zero detuning [215], and reads

$$
E_{\mathrm{R}}=\sqrt{4 g^{2}-\frac{\left(\Gamma_{\mathrm{pl}}-\Gamma_{\mathrm{ex}}\right)^{2}}{4}} .
$$

The criteria for strong coupling has been widely discussed in the literature [180, 195, 215,219], and, broadly speaking, it is defined when the rate of coherent energy exchange exceeds any damping mechanisms (e.g., $g \gg$ $\left.\Gamma_{\mathrm{pl}}, \Gamma_{\mathrm{ex}}\right)$. Although more than one criteria exists, a common approach to classify the strong coupling regime hinges on the fulfillment of the condition $E_{\mathrm{R}}>\left(\Gamma_{\mathrm{pl}}+\Gamma_{\mathrm{ex}}\right) / 2$ [provided that $\left.(4 g)^{2}>\left(\Gamma_{\mathrm{pl}}-\Gamma_{\mathrm{ex}}\right)^{2}\right][191,194,195,199,215]$. An alternative approach-which is particularly useful in the presence of large losses typical of plasmonic cavities-is to make use of the formalism introduced in Ref. [220], and define the following figure-of-merit (FOM):

$$
Q_{\mathrm{R}}=\frac{2 E_{\mathrm{R}}}{\Gamma_{\mathrm{pl}}+\Gamma_{\mathrm{ex}}} .
$$

As detailed in Ref. [220], this quantity is a quality factor that essentially quantifies the number of temporal oscillations in the occupation numbers of the new eigenstates. As such, $Q_{\mathrm{R}}=1$ unambiguously marks the onset of the strong-coupling regime: for $Q_{\mathrm{R}}<1$, that is, in the weak coupling regime, the original character of the modes is retained and the emitter population decays exponentially (Fig. 12c; top row); on the other hand, in the strong coupling regime, marked by $Q_{\mathrm{R}}>1$, the occupation numbers of the hybrid eigenstates oscillate in time with a characteristic Rabi-like frequency of $\Omega_{\mathrm{R}}=E_{\mathrm{R}} / \hbar$ (Fig. 12c; bottom row).

\subsection{Excitons in atomically-thin semiconductors}

In recent years, excitons-i.e., electron-hole pairs bounded through their mutual Coulomb interaction-in 2D materials like TMDCs have attracted a great deal of attention due to their singular optical properties [221-223]. These, to a great extent, reflect the presence of $2 \mathrm{D}$ excitons with large binding energies (hundreds of meV), thereby making such collective excitations stable even at room temperature. This is in stark contrast with excitons in conventional bulk 3D semiconductors which typically possess binding energies of the order of $k_{\mathrm{B}} T$ (where $k_{\mathrm{B}} T \simeq 26 \mathrm{meV}$ at $T=300 \mathrm{~K}$ ). The reason for this order-of-magnitude difference lies in inherently reduced screening in two dimensions [221,222, 224].

Furthermore, excitons in 2D TMDCs are not only stable at room temperature, but also host large (effective) dipole moments $\boldsymbol{\mu}$ [193] oriented in-plane. Note that both of these features are instrumental to achieve the strong-coupling regime at room temperature. 
As the low-energy physics of atomically-thin TMDCs is well-described by an effective Dirac-like Hamiltonian with finite mass [225,226], the valley degree of freedom can be used to manipulate the spin polarization of electrons-due to the sizable spin-orbit coupling exhibited by such materials [221] — using circularly polarized light [226-228]. Pioneering experiments have shown that it is possible to inject excitons with highly polarized spins that could be utilized for valleytronics [226-228]. Conversely, the valley polarization of these materials could also be exploited to coherently change the polarization state of the emitted light [229] or to steer the propagation direction of surface plasmon polaritons [230,231].

Moreover, the intrinsic properties of 2D TMDCs can be actively tuned by doping via electrostatic gating. Crucially, this not only modifies the occupation number of electrons (holes) in the conduction (valence) bands, but also strongly influences the excitonic spectrum owing to the change in the screening dynamics. Additionally, doped 2D TMDCs can also host charged excitons (dubbed trions); these are excitonic complexes formed when a neutral exciton binds to another electron or hole, thereby forming a charged threeparticle state [221]. These charged excitons are expected to play an important role in energy harvesting and in new optoelectronic devices [232].

At low temperatures and for small doping, biexcitons (four-body states) can be formed and contribute to the optical response of 2D TMDCs [233-235]. Decaying biexcitons in TMDCs could be an interesting platform to generate entangled pairs of photons for quantum technologies such as quantum networks and quantum cryptography. On a more fundamental viewpoint, when the density of excitons is very high, new states of electronic matter can be emerge such as dropletons [236] or Bose-Einstein condensates [181, 184, 237].

Most of the experimental works exploiting 2D TMDCs as platforms for studying light-matter interactions in the strong-coupling regime have predominantly involved $\mathrm{MoS}_{2}$, $\mathrm{WS}_{2}$, or $\mathrm{WSe}_{2}$, presumably, because they are commercially available, can be synthesized by CVD methods, and their optical properties are stable under ambient conditions over a long period of time. Nevertheless, with the advances in nanofabrication methods [238] enabling the possibility to realize new atomically-thin materials with new exotic properties, we anticipate the emergence of novel nanophotonic in the form of van der Waals heterostructures $[9,101]$ devices with functionalities going beyond the ones provided by the individual layers.

All the above-mentioned properties explain why 2D semiconducting TMDCs constitute exquisite architectures for exploring the rich physics of the strong-coupling regime (and, possibly, even in the ultrastrong-coupling regime [239]).

\subsection{Strong plasmon-exciton coupling with plasmonic cavities and TMDCs}

The formation of exciton polaritons created by exploiting excitons in layered TMDCs in combination with photonic or plasmonic cavities has now been demonstrated by many groups [34, 191, 193-200,209, 211,241], signaling that the field has now left its infant stage and has now reached adolescence. To illustrate this, in Fig. 13 we provide a cursory overview of a number of hybrid metal-TMDCs systems exhibiting strong plasmon-exciton interactions. In most cases, the experimental setup consists in hybrid systems composed by atomically-thin TMDCs in conjunction with plasmonic resonators, such as metallic nanoparticles with various shapes [193, 194, 197-199], nanoparticle-on-amirror (NPoM) geometries [196, 217, 242], plasmonic crystals [240,243], as well as plasmonic lattices [202,244-246]. A significant number of such plasmonic cavities are based on chemically grown metallic nanoparticles [247, 248]; this is motivated by the high-quality (up to the single-crystalline level) and extremely low surface roughness presented by these nanoparticles, which naturally yield plasmonic resonances with smaller linewidths.

Typically, in order to reach the so-called strong-coupling regime one tries to maximize the coupling strength $g$ while minimizing the overall damping mechanisms. As mentioned above, the latter can be achieved by employing highlycrystalline metal nanoparticles, whereas the former can be engineered by maximizing the spatial overlap of the plasmonic mode with the $2 \mathrm{D}$ material hosting the excitonic resonance. Lastly, excitonic resonances exhibiting large oscillator strengths are naturally better candidates as platforms for strong coupling (in this regard, $\mathrm{WS}_{2}$ seems to be particularly well-suited for that end [223]).

As portrayed in Fig. 13, substantial plasmon-exciton coupling has been achieved by combining different $2 \mathrm{D}$ TMDCs platforms with a plethora of plasmonic nanostructures such as nanorods, bipyramids, or nanodisks. Recently, it has been shown that one could improve the coupling strength (and thus obtain larger values of $E_{\mathrm{R}}$ ) by increasing the number of layers of TMDCs involved in the coupling process (Fig. 13d) [196, 198, 199]. Strikingly, the addition of a few more layers does not seem to significantly affect the excitonic resonance, despite modifying the material's bandstructure and creating an indirect band gap (instead the direct one exhibited in monolayers) [249]. Hence, in this spirit, varying the number of layers incrementally could be a viable strategy to study the weak-to-strong coupling transition.

In Table 3 we have summarized some of the latest works exploring strong coupling in hybrid metal-TMDCs systems. Even though the list is of course not exhaustive, it clearly shows a vast breath of different metal-TMDCs platforms suitable for investigating strong-coupling in nanophotonics. In the table, we provide the key parameters that characterize the plasmon-exciton interaction in each device, e.g., the amplitude of the measured Rabi-like splitting, along with the linewidths associated with the (uncoupled) plasmon and exciton resonances. These quantities then enable us to com- 

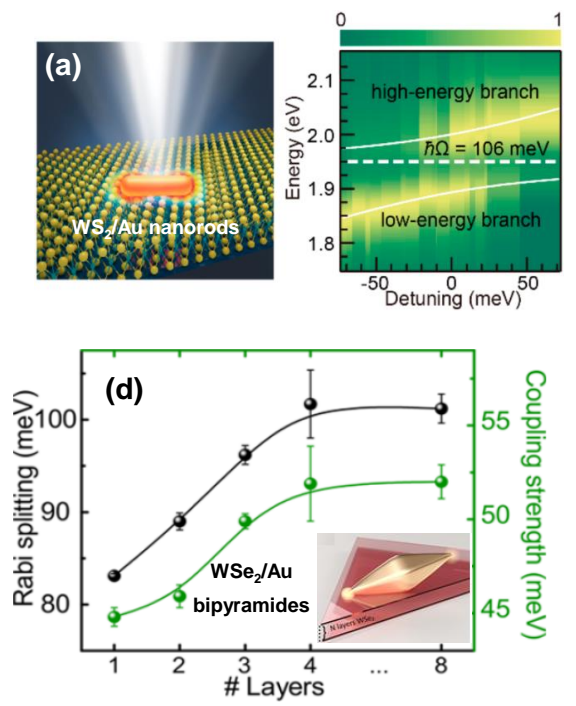

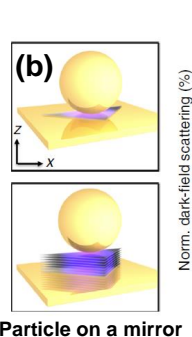

(e)

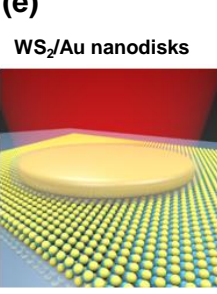



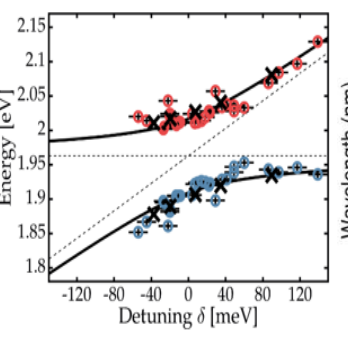

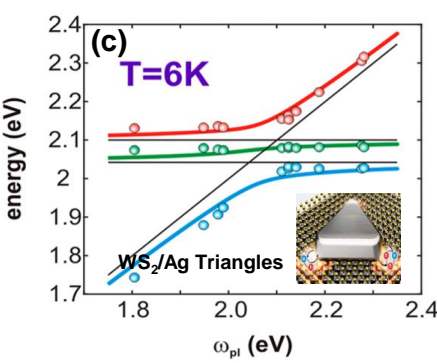

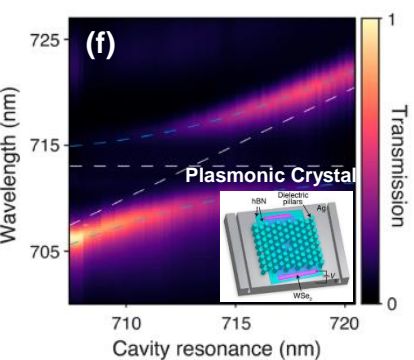

Figure 13 Overview of the different hybrid metal-TMDC strutures used in recent experiments. (a) Gold nanorods on WS 2 . Reproduced with permission. [193] Copyright 2017, American Chemical Society. (b) Metallic spheres-on-a-mirror with mono- and multilayer WSe $\mathrm{E}_{2}$ in the gap. Adapted with permission. [196] Copyright 2017, Springer Nature. (c) Plasmonic modes in silver nanotriangles coupled to excitons and trions in WS 2 . Reproduced with permission. [197] Copyright 2018, American Chemical Society. (d) Gold bypiramids on mono- and multilayer WSe 2 . Adapted with permission. [198] Copyright 2018, American Chemical Society. (e) Ultrathin gold nanodisks coupled to mono- and multilayer WS 2 . Reproduced with permission. [199] Copyright 2019, American Chemical Society. (f) Dielectrically loaded plasmonic crystal in monocrystalline surfaces coupled to $\mathrm{WSe}_{2}$. Adapted with permission. [240] Copyright 2019, American Chemical Society.

pute the FOM $Q_{\mathrm{R}}$ introduced in Eq. (27) that quantifies the coupling strength. Firstly, notice that the energy splittings can range from about $18 \mathrm{meV}$ [240] all the way up to values well above $150 \mathrm{meV}[199,217]$. In spite of this, most devices feature a FOM close to unity, i.e., $Q_{\mathrm{R}} \sim 1$, with many satisfy the strong coupling criterion $Q_{\mathrm{R}}>1$ (even though by a relatively small margin). Importantly, it is clear that the FOM can increase significantly when considering few-layer TMDCs (as opposed to its single-layer version) [198, 199].

For comparison purposes with other platforms capable of achieving strong coupling at room temperature, we have also included in Table 3 the different parameters reported by Wersäll et al. [211] using LSPRs coupled to organic molecules as the "excitonic material". Remarkably, they reach a FOM of 3.2, which constitutes the highest value reported so far for single plasmonic resonators coupled to emitters. This exceptionally large value could be explained by the fact that the J-aggregates used in this experiment covers the whole silver nanotriangles, therefore maximizing the overlap of the plasmon mode with the exciton-supporting. This is in contrast with the TMDCs-based setups that have been explored so far, where the TMDCs layer interacts mostly with only one fraction of the plasmon mode. Hence, we speculate on the possibility of increasing the overall coupling strengh by, say, encapsulating such metallic nanoparticles with 2D TMDCs.

\subsection{Perspectives on strong coupling}

Since the first demonstration of strong coupling between LSPRs in metallic nanoparticles and excitons in 2D TMDCs, a growing number of experimental works have reported signatures of plasmon-exciton strong coupling probed by optical measurements. From the point of view of the characterization methods, many of the aforementioned experiments have used dark-field scattering spectroscopy methods [193, 194, 198, 199] for determining the amplitude of the polariton anti-crossing and infer from it wether the interaction is in the weak or strong coupling regime. However, as it has been recently pointed out by a number of theoretical [219, 250] and experimental [203] works, the sole measurement of the scattering cross-section is not sufficient to unambiguously conclude if the system is in within strongcoupling regime. Indeed, the splitting observed in scattering measurements could simply be a consequence of plasmonenhanced absorption or or more Fano-type physics [250]. Therefore, it is imperative to use other complementary characterization methods that can provide further insight about the plasmon-exciton coupling mechanism at work. For instance, examples of such methods are photoluminescence in the far- [203,211, 217, 251,252] and near-field [205, 206] and/or spectroscopic methods enabling direct or indirect access to the absorption cross-section [199, 250]. In addition, plasmon-exciton polaritons have been observed using electron energy-loss-spectroscopy [200]; this technique opens 


\begin{tabular}{ccccccccc}
\hline References & LSPR & TMDC & \# of layers & $E_{\mathrm{R}}[\mathrm{meV}]$ & $\Gamma_{\mathrm{pl}}[\mathrm{meV}]$ & $\Gamma_{\mathrm{ex}}[\mathrm{meV}]$ & $Q_{\mathrm{R}}$ & Temp. [K] \\
\hline \hline$[194]$ & Ag nanorod & $\mathrm{WSe}_{2}$ & 1 & 49.5 & 98 & 43 & 0.7 & $\mathrm{RT}$ \\
{$[193]$} & Au nanorods & $\mathrm{WS}_{2}$ & 1 & 106 & 149 & 57 & 1.03 & $\mathrm{RT}$ \\
{$[196]$} & SoM & $\mathrm{WSe}_{2}$ & 12 & 140 & 120 & 70 & 1.47 & $\mathrm{RT}$ \\
{$[197]$} & Ag nanotriangles & $\mathrm{WS}_{2}$ & 1 & 120 & 190 & 20 & 1.14 & $\mathrm{RT} / 6 \mathrm{~K}$ \\
{$[198]$} & Au bipyramids & $\mathrm{WSe}_{2}$ & 8 & 101 & 110 & 63 & 1.17 & $\mathrm{RT}$ \\
{$[242]$} & NCoM & $\mathrm{WS}_{2}$ & 1 & 145 & 220 & 50 & 1.07 & $\mathrm{RT}$ \\
{$[243]$} & Au nanoholes & $\mathrm{WS}_{2}$ & 1 & 162 & 380 & 60 & 0.74 & $\mathrm{RT}$ \\
{$[199]$} & Au nanodisks & $\mathrm{WS}_{2}$ & 7 & 175 & 170 & 44 & 1.64 & $\mathrm{RT}$ \\
{$[240]$} & Ag DLPC & $\mathrm{WSe}_{2}$ & 1 & 18.2 & 7 & 7.5 & 2.51 & $4 \mathrm{~K}$ \\
{$[217]$} & Au NCoM & $\mathrm{MoS}_{2}$ & 1 & 190 & 280 & 50 & 1.15 & RT \\
\hline$[211]$ & Ag nanotriangles & J-aggregates & N. A. & 400 & 150 & 100 & 3.2 & RT \\
\hline
\end{tabular}

Table 3 Reported parameters from experimental works on individual localized surface plasmon resonances (LSPRs) interaction with atomically-thin ransition metal dichalcogenides (TMDCs) and corresponding calculated figure-of-merit (FOM) $Q_{\mathrm{R}}$. For comparison parameters and FOM from the experimental work of Wersäll et al. [211] with silver nanotriangles coupled to organic molecules are reported in the last columns. Abbreviations: sphere-on-a-Mirror (SoM), nanocube-on-a-mirror (NCoM), room-temperature (RT), dielectrically loaded plasmonic cavity (DLPC).

enticing perspectives for probing plasmon-exciton interactions at the true nanometer scale. Furthermore, recent experimental works involving phololuminescence spectroscopy of LSPR coupled to organic molecules [251] or excitons in TMDCs [196, 217,252] have reported that most of the spontaneously emitted light originates from the LPB, with very little emission arising from the UPB. Nevertheless, more investigations-e.g., using time-domain photoluminescence spectroscopy - are needed in order to obtain a deeper understanding of the dynamics associated with the UPB and the LPB in these systems.

Moreover, from the point of view of methods for fabricating $2 \mathrm{D}$ materials, there are many prospective systems waiting to be explored [238]. On the other hand, the recent discovery of superconductivy in graphene by simply twisting adjacent layers in bilayer graphene [7] hints at the possibility of tailoring many-body interactions involved in exciton formation by twisting TMDCs (dubbed "Moiré excitons") [253-256]. This new degree of freedom, which is not present in conventional semiconductors, opens the possibility to design novel exciton species that may in principle be optimally tailored in order to maximize the coupling with electromagnetic modes.

Finally, in order to be suitable for quantum-based applications, strong coupling achieved in hybrid metal-TMDC systems must necessarily pass a few experimental tests and demonstrate quantum coherence in the coupling between plasmon and excitons. In this regards, direct measurement in the time-domain of Rabi oscillations [257] would be a decisive towards that end (though experimentally challenging due to the fast rate of oscillation), as well as measuring the statistics and correlation functions of the photons emitted from these systems. Theoretical [258, 259] and experimental [260] works have paved the way in that direction for systems involving organic molecules interacting with LSPRs and still need to be demonstrated for 2D material-based devices.

\section{Outlook}

The advent of atomically-thin materials - initiated and spearheaded by graphene-has opened enticing new routes for nanophotonics in two dimensions and ensuing nanostructures. In this Review, we provide a hopefully representative sample of a breadth of polaritons and related light-matter interactions enabled by $2 \mathrm{D}$ polaritons (e.g., plasmon polaritons, exciton polaritons, phonon polaritons, etc). Despite the fast-paced growth we have been witnessing over the last couple of years, it is not unfathomable to think that research in 2D nanophotonics has only scratched the surface, with a plethora of exciting possibilities over the horizon. While some of these possibilities are yet to be recognized, new avenues are already starting to be pursued. Examples of the latter are hyperbolic [36,261] and chiral polaritonics [231,262], using 2D polaritons for controlling the dynamics of emitters placed in the vicinity of a 2D material [263], or the engineering of new flavors of polaritons $[29,30]$ (e.g., plasmonexciton polaritons, plasmon-phonon polaritons, etc). Other alluring research directions are 2D material-based topological photonics [264,265], twist-polaritonics [266-268], as well as transdimensional photonics [269] (i.e., the intermediate regime between the "strictly 3D" and the "strictly 2D" regime).

The strong field confinement and high tunability provided by $2 \mathrm{D}$ polaritons makes them an outstanding tool to control and study light-matter interactions at the nanoscale. In the light of the continuously increasing library of 2D materials with many different properties, we anticipate that research in "flatland nanophotonics" will continue to blossom in the coming years, potentially leading to novel nanoscale optoelectronic devices. 
Acknowledgements. The Center for Nanostructured Graphene is sponsored by the Danish National Research Foundation (Project No. DNRF103). S. X. is supported by the Independent Research Fund Denmark (Project No. 9041-00333B). The Center for Nano Optics is supported by the University of Southern Denmark (SDU 2020 funding). J. D. C. and N. A. M. are supported by VILLUM Fonden (Grant No. 16498).

Key words: Polaritons, plasmons, excitons, light-matter interaction, 2D materials, nanophotonics
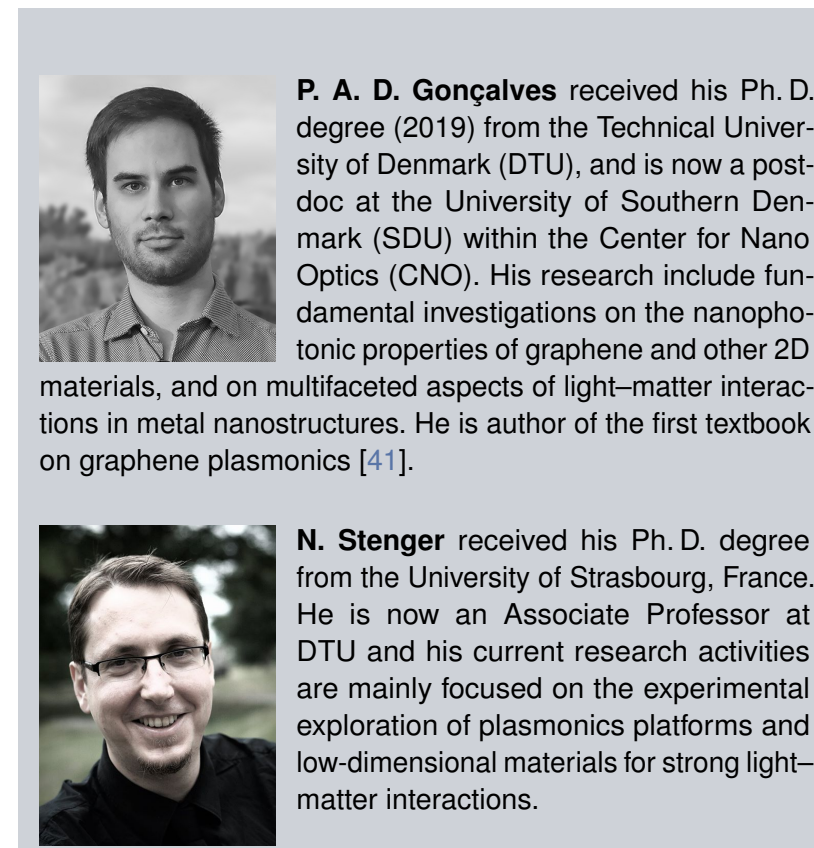

N. Stenger received his Ph. D. degree from the University of Strasbourg, France. $\mathrm{He}$ is now an Associate Professor at DTU and his current research activities are mainly focused on the experimental exploration of plasmonics platforms and low-dimensional materials for strong lightmatter interactions.

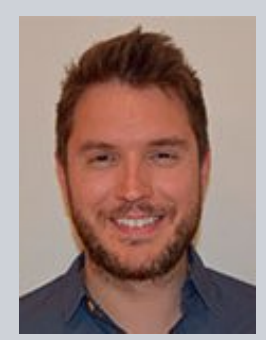

J. D. Cox received his Ph. D. degree (2013) from the University of Western Ontario, Canada. He is an Assistant Professor in the SDU CNO and the Danish Institute for Advanced Study (D-IAS). His interests include nanophotonics, nonlinear and quantum optics, and condensed matter physics.

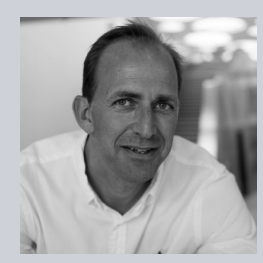

N. A. Mortensen received his Ph. D. (2001) and Dr. Techn. (2006) degrees from DTU. He is a professor and VILLUM Investigator at SDU CNO, and a D-IAS Chair of Technical Science. He focuses on quantum and nonlocal light-matter interactions.

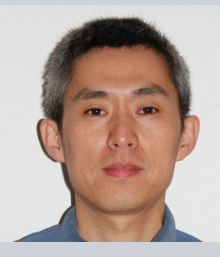

Sanshui Xiao received his Ph. D. degree (2004) at Zhejiang University. He joined DTU in 2006 and now is an Associate Professor at DTU. His current research interests focus on light-matter interactions in nanostructures and their applications in optoelectronic devices.

\section{References}

[1] K. S. Novoselov, A. K. Geim, S. V. Morozov, D. Jiang, Y. Zhang, S. V. Dubonos, I. V. Grigorieva, and A. A. Firsov. Science 2004, 306, 666.

[2] K. S. Novoselov, D. Jiang, F. Schedin, T. J. Booth, V. V. Khotkevich, S. V. Morozov, and A. K. Geim. Proc. Natl. Acad. Sci. U. S. A. 2005, 102, 10451.

[3] S. Tang, H. Wang, H. S. Wang, Q. Sun, X. Zhang, C. Cong, H. Xie, X. Liu, X. Zhou, F. Huang, X. Chen, T. Yu, F. Ding, X. Xie, and M. Jiang. Nat. Commun. 2015, 6, 6499.

[4] X. Xu, Z. Zhang, L. Qiu, J. Zhuang, L. Zhang, H. Wang, C. Liao, H. Song, R. Qiao, P. Gao, Z. Hu, L. Liao, Z. Liao, D. Yu, E. Wang, F. Ding, H. Peng, and K. Liu. Nat. Nanotechnol. 2016, 11, 930.

[5] T. Wu, X. Zhang, Q. Yuan, J. Xue, G. Lu, Z. Liu, H. Wang, H. Wang, F. Ding, Q. Yu, X. Xie, and M. Jiang. Nat. Mater. 2016, 15, 43.

[6] X. Duan, C. Wang, J. C. Shaw, R. Cheng, Y. Chen, H. Li, X. Wu, Y. Tang, Q. Zhang, A. Pan, J. Jiang, R. Yu, Y. Huang, and X. Duan. Nat. Nanotechnol. 2014, 9, 1024.

[7] Y. Cao, V. Fatemi, S. Fang, K. Watanabe, T. Taniguchi, E. Kaxiras, and P. Jarillo-Herrero. Nature 2018, 556, 43.

[8] Y. Cao, V. Fatemi, A. Demir, S. Fang, S. L. Tomarken, J. Y. Luo, J. D. Sanchez-Yamagishi, K. Watanabe, T. Taniguchi, E. Kaxiras, R. C. Ashoori, and P. Jarillo-Herrero. Nature 2018, 556, 80.

[9] K. S. Novoselov, A. Mishchenko, A. Carvalho, and A. H. Castro Neto. Science 2016, 353, aac9439.

[10] F. Pizzocchero, L. Gammelgaard, B. S. Jessen, J. M. Caridad, L. Wang, J. Hone, P. Bøggild, and T. J. Booth. Nat. Commun. 2016, 7, 11894.

[11] X. Yang, C. Cheng, Y. Wang, L. Qiu, and D. Li. Science 2013, 341, 534.

[12] W. Wu, L. Wang, Y. Li, F. Zhang, L. Lin, S. Niu, D. Chenet, X. Zhang, Y. Hao, T. F. Heinz, J. Hone, and Z. L. Wang. Nature 2014, 514, 470.

[13] M. Zhao, J. Zhang, N. Gao, P. Song, M. Bosman, B. Peng, B. Sun, C.-W. Qiu, Q.-H. Xu, Q. Bao, and K. P. Loh. Adv. Mater. 2016, 28, 3138.

[14] B. Anasori, M. R. Lukatskaya, and Y. Gogotsi. Nat. Rev. Mater. 2017, 2, 16098.

[15] F. Xia, H. Wang, D. Xiao, M. Dubey, and A. Ramasubramaniam. Nat. Photonics 2014, 8, 899.

[16] F. Bonaccorso, Z. Sun, T. Hasan, and A. C. Ferrari. Nat. Photonics 2010, 4, 611.

[17] M. Liu, X. Yin, E. Ulin-Avila, B. Geng, T. Zentgraf, L. Ju, F. Wang, and X. Zhang. Nature 2011, 474, 64.

[18] C. T. Phare, Y.-H. D. Lee, J. Cardenas, and M. Lipson. Nat. Photonics 2015, 9, 511.

[19] Y. Ding, X. Zhu, S. Xiao, H. Hu, L. H. Frandsen, N. A. Mortensen, and K. Yvind. Nano Lett. 2015, 15, 4393.

[20] T. Mueller, F. Xia, and P. Avouris. Nat. Photonics 2010, 4, 297.

[21] Y. Ding, X. Guan, X. Zhu, H. Hu, S. I. Bozhevolnyi, L. K. Oxenlowe, K. J. Jin, N. A. Mortensen, and S. Xiao. Nanoscale 2017, 9, 15576.

[22] Y. Ding, Z. Chen, X. Zhu, K. Yvind, J. Dong, M. Galili, H. Hu, N. A. Mortensen, S. Xiao, and L. K. Oxenløwe. Nanophotonics 2019, in press. Doi: 10.1515/nanoph-20190167.

[23] D. Rodrigo, O. Limaj, D. Janner, D. Etezadi, F. J. García de Abajo, V. Pruneri, and H. Altug. Science 2015, 349, 165. 
[24] D. Rodrigo, A. Tittl, O. Limaj, F. J. García de Abajo, V. Pruneri, and H. Altug. Light Sci. Appl. 2017, 6, e16277.

[25] N. Dontschuk, A. Stacey, A. Tadich, K. J. Rietwyk, A. Schenk, M. T. Edmonds, O. Shimoni, C. I. Pakes, S. Prawer, and J. Cervenka. Nat. Commun. 2015, 6, 6563.

[26] H. Hu, X. Yang, F. Zhai, D. Hu, R. Liu, K. Liu, Z. Sun, and Q. Dai. Nat. Commun. 2016, 7, 12334.

[27] X. Chen, Y. J. Park, M. Kang, S.-K. Kang, J. Koo, S. M. Shinde, J. Shin, S. Jeon, G. Park, Y. Yan, M. R. MacEwan, W. Z. Ray, K.-M. Lee, J. A. Rogers, and J.-H. Ahn. Nat. Commun. 2018, 9, 1690.

[28] H. Hu, X. Yang, X. Guo, K. Khaliji, S. R. Biswas, F. J. García de Abajo, T. Low, Z. Sun, and Q. Dai. Nat. Commun. 2019, 10, 1131.

[29] D. N. Basov, M. M. Fogler, and F. J. García de Abajo. Science 2016, 354, aag1992.

[30] T. Low, A. Chaves, J. D. Caldwell, A. Kumar, N. X. Fang, P. Avouris, T. F. Heinz, F. Guinea, L. Martin-Moreno, and F. Koppens. Nat. Mater. 2017, 16, 182.

[31] A. A. Balandin and D. L. Nika. Mater. Today 2012, 15, 266.

[32] F. H. L. Koppens, D. E. Chang, and F. J. García de Abajo. Nano Lett. 2011, 11, 3370.

[33] L. Britnell, R. M. Ribeiro, A. Eckmann, R. Jalil, B. D. Belle, A. Mishchenko, Y.-J. Kim, R. V. Gorbachev, T. Georgiou, S. V. Morozov, A. N. Grigorenko, A. K. Geim, C. Casiraghi, A. H. Castro Neto, and K. S. Novoselov. Science 2013, 340 , 1311.

[34] X. Liu, T. Galfsky, Z. Sun, F. Xia, E.-c. Lin, Y.-H. Lee, S. Kéna-Cohen, and V. M. Menon. Nat. Photonics 2015, 9, 30.

[35] E. Yoxall, M. Schnell, A. Y. Nikitin, O. Txoperena, A. Woessner, M. B. Lundeberg, F. Casanova, L. E. Hueso, F. H. L. Koppens, and R. Hillenbrand. Nat. Photonics 2015, 9, 674.

[36] S. Dai, Z. Fei, Q. Ma, A. S. Rodin, M. Wagner, A. S. McLeod, M. K. Liu, W. Gannett, W. Regan, K. Watanabe, T. Taniguchi, M. Thiemens, G. Dominguez, A. H. Castro Neto, A. Zettl, F. Keilmann, P. Jarillo-Herrero, M. M. Fogler, and D. N. Basov. Science 2014, 343, 1125.

[37] P. Li, M. Lewin, A. V. Kretinin, J. D. Caldwell, K. S. Novoselov, T. Taniguchi, K. Watanabe, F. Gaussmann, and T. Taubner. Nat. Commun. 2015, 6, 7507.

[38] A. Woessner, M. B. Lundeberg, Y. Gao, A. Principi, P. Alonso-Gonzlez, M. Carrega, K. Watanabe, T. Taniguchi, G. Vignale, M. Polini, J. Hone, R. Hillenbrand, and F. H. L. Koppens. Nat. Mater. 2015, 14, 421.

[39] M. Polini and F. H. L. Koppens. Nat. Mater. 2015, 14, 1187

[40] W. Ma, P. Alonso-Gonzalez, S. Li, A. Y. Nikitin, J. Yuan, J. Martin-Sanchez, J. Taboada-Gutierrez, I. Amenabar, P. Li, S. Velez, C. Tollan, Z. Dai, Y. Zhang, S. Sriram, K. KalantarZadeh, S.-T. Lee, R. Hillenbrand, and Q. Bao. Nature 2018, 562, 557.

[41] P. A. D. Gonçalves and N. M. R. Peres, An Introduction to Graphene Plasmonics 2016 (World Scientific, Singapore).

[42] F. J. García de Abajo. ACS Photonics 2014, 1, 135.

[43] G. X. Ni, A. S. McLeod, Z. Sun, L. Wang, L. Xiong, K. W. Post, S. S. Sunku, B.-Y. Jiang, J. Hone, C. R. Dean, M. M. Fogler, and D. N. Basov. Nature 2018, 557, 530.

[44] S. Xiao, X. Zhu, B.-H. Li, and N. A. Mortensen. Front. Phys. 2016, 11, 117801

[45] A. H. Castro Neto, F. Guinea, N. M. R. Peres, K. S. Novoselov, and A. K. Geim. Rev. Mod. Phys. 2009, 81, 109.
[46] L. Kong, B. Yan, and X. Hu. Plasma Sci. Technol. 2007, 9 , 519.

[47] D. G. Papageorgiou, I. A. Kinloch, and R. J. Young. Prog Mater. Sci. 2017, 90, 75.

[48] T. Das, B. K. Sharma, A. K. Katiyar, and J.-H. Ahn. J. Semicond. 2018, 39, 011007.

[49] The relation between graphene's Fermi energy, $E_{F}$, and its electronic density, $n_{e}$, is given by $E_{F}=\hbar v_{F} \sqrt{\pi n_{e}}$. Typical values for the carrier density in doped graphene are $n_{e}=$ $10^{11}-10^{13} \mathrm{~cm}^{-2}$ [50-52], though values as high as $n_{e}=$ $4 \times 10^{14} \mathrm{~cm}^{-2}$ have been reported [270].

[50] L. Ju, B. Geng, J. Horng, C. Girit, M. Martin, Z. Hao, H. A. Bechtel, X. Liang, A. Zettl, Y. R. Shen, and F. Wang. Nat. Nanotechnol. 2011, 6, 630.

[51] Z. Fei, A. S. Rodin, G. O. Andreev, W. Bao, A. S. McLeod, M. Wagner, L. M. Zhang, Z. Zhao, M. Thiemens, G. Dominguez, M. M. Fogler, A. H. C. Neto, C. N. Lau, F. Keilmann, and D. N. Basov. Nature 2012, 487, 82.

[52] J. Chen, M. Badioli, P. Alonso-Gonzlez, S. Thongrattanasiri, F. Huth, J. Osmond, M. Spasenovi, A. Centeno, A. Pesquera, P. Godignon, A. Z. Elorza, N. Camara, F. J. García de Abajo, R. Hillenbrand, and F. H. L. Koppens. Nature 2012, 487, 77.

[53] H. Liu, Y. Liu, and D. Zhu. J. Mater. Chem. 2011, 21, 3335.

[54] At the time of writing, several hundreds of 2D materials have been identified. Most of them and their properties can be found in "2D materials databases" such as the ones provided in Refs. $[55,56]$.

[55] S. Haastrup, M. Strange, M. Pandey, T. Deilmann, P. S. Schmidt, N. F. Hinsche, M. N. Gjerding, D. Torelli, P. M. Larsen, A. C. Riis-Jensen, J. Gath, K. W. Jacobsen, J. J. Mortensen, T. Olsen, and K. S. Thygesen. 2D Mater. 2018, 5,042002 .

[56] N. Mounet, M. Gibertini, P. Schwaller, D. Campi, A. Merkys, A. Marrazzo, T. Sohier, I. E. Castelli, A. Cepellotti, G. Pizzi, and N. Marzari. Nat. Nanotechnol. 2018, 13, 246.

[57] N. D. Mermin. Phys. Rev. B 1970, 1, 2362.

[58] J. Horng, C.-F. Chen, B. Geng, C. Girit, Y. Zhang, Z. Hao, H. A. Bechtel, M. Martin, A. Zettl, M. F. Crommie, Y. R. Shen, and F. Wang. Phys. Rev. B 2011, 83, 165113.

[59] L. A. Falkovsky and A. A. Varlamov. Eur. Phys. J. B 2007, 56, 281.

[60] L. A. Falkovsky and S. S. Pershoguba. Phys. Rev. B 2007, $76,153410$.

[61] B. Wunsch, T. Stauber, F. Sols, and F. Guinea. New J. Phys. 2006, 8, 318 .

[62] E. H. Hwang and S. Das Sarma. Phys. Rev. B 2007, 75, 205418.

[63] P. A. D. Gonçalves, E. J. C. Dias, S. Xiao, M. I. Vasilevskiy, N. A. Mortensen, and N. M. R. Peres. ACS Photonics 2016, 3, 2176.

[64] P. A. D. Gonçalves, S. I. Bozhevolnyi, N. A. Mortensen, and N. M. R. Peres. Optica 2017, 4, 595.

[65] P. A. D. Gonçalves, S. Xiao, N. M. R. Peres, and N. A. Mortensen. ACS Photonics 2017, 4, 3045.

[66] R. Yu, J. D. Cox, J. R. M. Saavedra, and F. J. García de Abajo. ACS Photonics 2017, 4, 3106.

[67] M. B. Lundeberg, Y. Gao, R. Asgari, C. Tan, B. Van Duppen, M. Autore, P. Alonso-González, A. Woessner, K. Watanabe, T. Taniguchi, R. Hillenbrand, J. Hone, M. Polini, and F. H. L. Koppens. Science 2017, 357, 187. 
[68] F. Stern. Phys. Rev. Lett. 1967, 18, 546.

[69] P. A. D. Gonçalves, E. J. C. Dias, Y. V. Bludov, and N. M. R. Peres. Phys. Rev. B 2016, 94, 195421.

[70] J. Christensen, A. Manjavacas, S. Thongrattanasiri, F. H. L. Koppens, and F. J. García de Abajo. ACS Nano 2012, 6, 431.

[71] H. Yan, T. Low, W. Zhu, Y. Wu, M. Freitag, X. Li, F. Guinea, P. Avouris, and F. Xia. Nat. Photonics 2013, 7, 394.

[72] I. J. Luxmoore, C. H. Gan, P. Q. Liu, F. Valmorra, P. Li, J. Faist, and G. R. Nash. ACS Photonics 2014, 1, 1151.

[73] C. Sorger, S. Preu, J. Schmidt, S. Winnerl, Y. V. Bludov, N. M. R. Peres, M. I. Vasilevskiy, and H. B. Weber. New J. Phys. 2015, 17, 053045.

[74] D. B. Farmer, P. Avouris, Y. Li, T. F. Heinz, and S.-J. Han. ACS Photonics 2016, 3, 553.

[75] X. Zhu, W. Yan, N. A. Mortensen, and S. Xiao. Opt. Express 2013, 21, 3486.

[76] Y. Dai, X. Zhu, N. A. Mortensen, J. Zi, and S. Xiao. J. Opt. 2015, 17, 065002 .

[77] H. Yan, X. Li, B. Chandra, G. Tulevski, Y. Wu, M. Freitag, W. Zhu, P. Avouris, and F. Xia. Nat. Nanotechnol. 2012, 7 , 330.

[78] H. Yan, F. Xia, Z. Li, and P. Avouris. New J. Phys. 2012, 14, 125001.

[79] S. Thongrattanasiri, A. Manjavacas, and F. J. García de Abajo. ACS Nano 2012, 6, 1766.

[80] Z. Fang, S. Thongrattanasiri, A. Schlather, Z. Liu, L. Ma, Y. Wang, P. M. Ajayan, P. Nordlander, N. J. Halas, and F. J. García de Abajo. ACS Nano 2013, 7, 2388.

[81] Z. Fang, Y. Wang, A. E. Schlather, Z. Liu, P. M. Ajayan, F. J. García de Abajo, P. Nordlander, X. Zhu, and N. J. Halas. Nano Lett. 2014, 14, 299.

[82] X. Zhu, W. Wang, W. Yan, M. B. Larsen, P. Bøggild, T. G. Pedersen, S. Xiao, J. Zi, and N. A. Mortensen. Nano Lett. 2014, 14, 2907.

[83] Z. Wang, T. Li, K. Almdal, N. A. Mortensen, S. Xiao, and S. Ndoni. Opt. Lett. 2016, 41, 5345.

[84] W. Wang, S. Xiao, and N. A. Mortensen. Phys. Rev. B 2016, 93, 165407.

[85] J. D. Cox and F. J. García de Abajo. Nat. Commun. 2014, 5,5725 .

[86] W. Wang, T. Christensen, A.-P. Jauho, K. S. Thygesen, M. Wubs, and N. A. Mortensen. Sci. Rep. 2015, 5, 9535.

[87] K. Y. M. Yeung, J. Chee, H. Yoon, Y. Song, J. Kong, and D. Ham. Nano Lett. 2014, 14, 2479.

[88] P. Q. Liu, F. Valmorra, C. Maissen, and J. Faist. Optica 2015, 2, 135.

[89] A. L. Fetter. Phys. Rev. B 1986, 33, 3717.

[90] A. L. Fetter. Phys. Rev. B 1986, 33, 5221.

[91] X. Xia and J. J. Quinn. Phys. Rev. B 1994, 50, 8032.

[92] W. Wang, P. Apell, and J. Kinaret. Phys. Rev. B 2011, 84, 085423.

[93] W. Wang, S. P. Apell, and J. M. Kinaret. Phys. Rev. B 2012, $86,125450$.

[94] W. Wang. J. Phys.: Cond. Matter 2012, 24, 402202.

[95] M. Abramowitz and I. A. Stegun, Handbook of mathematical functions: with formulas, graphs, and mathematical tables 1972 (Dover, New York), also freely available at: http://people.math.sfu.ca/ cbm/aands/.

[96] V. A. Volkov and S. A. Mikhailov. Sov. Phys. JETP 1988, 67, 1639.
[97] This fact was noted by Fetter in Ref. [90], where he used the same method for expanding the charge density instead of expanding the potential. Naturally, such a formulation is tantamount - and ultimately equivalent- to the one described here. In any case, and perhaps more important for practical matters, we stress that the approach we follow here also admits a matrix with analytical matrix elements.

[98] S. Thongrattanasiri, F. H. L. Koppens, and F. J. García de Abajo. Phys. Rev. Lett. 2012, 108, 047401.

[99] T. Christensen, W. Wang, A.-P. Jauho, M. Wubs, and N. A. Mortensen. Phys. Rev. B 2014, 90, 241414.

[100] K. O. Wedel, N. A. Mortensen, K. S. Thygesen, and M. Wubs. Phys. Rev. B 2018, 98, 155412.

[101] A. K. Geim and I. V. Grigorieva. Nature 2013, 499, 419.

[102] P. Alonso-Gonzlez, A. Y. Nikitin, Y. Gao, A. Woessner, M. B. Lundeberg, A. Principi, N. Forcellini, W. Yan, S. Vlez, A. J. Huber, K. Watanabe, T. Taniguchi, F. Casanova, L. E. Hueso, M. Polini, J. Hone, F. H. L. Koppens, and R. Hillenbrand. Nat. Nanotechnol. 2017, 12, 31.

[103] D. Alcaraz Iranzo, S. Nanot, E. J. C. Dias, I. Epstein, C. Peng, D. K. Efetov, M. B. Lundeberg, R. Parret, J. Osmond, J.-Y. Hong, J. Kong, D. R. Englund, N. M. R. Peres, and F. H. L. Koppens. Science 2018, 360, 291.

[104] E. J. C. Dias, D. A. Iranzo, P. A. D. Gonçalves, Y. Hajati, Y. V. Bludov, A.-P. Jauho, N. A. Mortensen, F. H. L. Koppens, and N. M. R. Peres. Phys. Rev. B 2018, 97, 245405.

[105] A. R. Echarri, J. D. Cox, and F. J. García de Abajo. Optica 2019, 6, 630.

[106] S. Raza, S. I. Bozhevolnyi, M. Wubs, and N. A. Mortensen. J. Phys.: Condens. Matter 2015, 27, 183204.

[107] C. Ciracì, R. T. Hill, J. J. Mock, Y. Urzhumov, A. I. Fernández-Domínguez, S. A. Maier, J. B. Pendry, A. Chilkoti, and D. R. Smith. Science 2012, 337, 1072.

[108] P. J. Feibelman. Prog. Surf. Sci. 1982, 12, 287.

[109] T. Christensen, W. Yan, A.-P. Jauho, M. Soljačić, and N. A. Mortensen. Phys. Rev. Lett. 2017, 118, 157402.

[110] P. A. D. Gonçalves, T. Christensen, N. Rivera, A.-P. Jauho, N. A. Mortensen, and M. Soljai. arXiv:1904.09279 2019,

[111] P. Ginzburg and A. V. Zayats. ACS Nano 2013, 7, 4334.

[112] N. A. Mortensen. Photonics Nanostructures: Fundam. Appl. 2013, 11, 303.

[113] T. V. Teperik, P. Nordlander, J. Aizpurua, and A. G. Borisov. Phys. Rev. Lett. 2013, 110, 263901.

[114] A. I. Fernández-Domínguez, P. Zhang, Y. Luo, S. A. Maier, F. J. García-Vidal, and J. B. Pendry. Phys. Rev. B 2012, 86, 241110.

[115] W. Zhu, R. Esteban, A. G. Borisov, J. J. Baumberg, P. Nordlander, H. J. Lezec, J. Aizpurua, and K. B. Crozier. Nat. Commun. 2016, 7, 11495.

[116] G. Toscano, J. Straubel, A. Kwiatkowski, C. Rockstuhl, F. Evers, H. Xu, N. A. Mortensen, and M. Wubs. Nat. Commun. 2015, 6, 7132.

[117] R. W. Boyd, Nonlinear optics 2008 (Academic Press, Amsterdam).

[118] E. Garmire. Opt. Express 2013, 21, 30532.

[119] M. Kauranen and A. V. Zayats. Nat. Photonics 2012, 6, 737.

[120] C. Voisin, N. Del Fatti, D. Christofilos, and F. Vallée. J. Phys. Chem. B 2001, 105, 2264.

[121] M. Abb, P. Albella, J. Aizpurua, and O. L. Muskens. Nano Lett. 2011, 11, 2457.

[122] S. Palomba and L. Novotny. Phys. Rev. Lett. 2008, 101, 056802. 
[123] H. Harutyunyan, G. Volpe, R. Quidant, and L. Novotny. Phys. Rev. Lett. 2012, 108, 217403.

[124] Y. Zhang, F. Wen, Y.-R. Zhen, P. Nordlander, and N. J. Halas. Proc. Natl. Acad. Sci. U.S.A. 2013, 110, 9215.

[125] J. Butet, P.-F. Brevet, and O. J. F. Martin. ACS Nano 2015, 9, 10545.

[126] A. Krasnok, M. Tymchenko, and A. Alù. Mater. Today 2018, 21,8 .

[127] J. D. Cox, A. Marini, and F. J. García de Abajo. Nat. Commun. 2017, 8, 14380.

[128] S. A. Mikhailov. Phys. Rev. B 2011, 84, 045432.

[129] A. V. Gorbach. Phys. Rev. A 2013, 87, 013830.

[130] M. Gullans, D. E. Chang, F. H. L. Koppens, F. J. García de Abajo, and M. D. Lukin. Phys. Rev. Lett. 2013, 111, 247401.

[131] X. Yao, M. Tokman, and A. Belyanin. Phys. Rev. Lett. 2014, 112,055501 .

[132] J. Tao, Z. Dong, J. K. W. Yang, and Q. J. Wang. Opt. Express 2015, 23, 7809 .

[133] J. D. Cox and F. J. García de Abajo. ACS Photonics 2015, 2, 306.

[134] M. Jablan and D. E. Chang. Phys. Rev. Lett. 2015, 114, 236801.

[135] T. Christensen, W. Yan, A.-P. Jauho, M. Wubs, and N. A. Mortensen. Phys. Rev. B 2015, 92, 121407(R).

[136] M. T. Manzoni, I. Silveiro, F. J. García de Abajo, and D. E. Chang. New J. Phys. 2015, 17, 083031.

[137] T. J. Constant, S. M. Hornett, D. E. Chang, and E. Hendry. Nature Phys. 2016, 12, 124.

[138] M. M. Jadidi, J. C. König-Otto, S. Winnerl, A. B. Sushkov, H. D. Drew, T. E. Murphy, and M. Mittendorff. Nano Lett. 2016, 16, 2734.

[139] J. D. Cox, I. Silveiro, and F. J. García de Abajo. ACS Nano 2016, 10, 1995.

[140] H. Rostami, M. I. Katsnelson, and M. Polini1. Phys. Rev. B 2017, 95, 035416.

[141] J. D. Cox, R. Yu, and F. J. García de Abajo. Phys. Rev. B 2017, 96, 045442.

[142] D. Kundys, B. V. Duppen, O. P. Marshall, F. Rodriguez, I. Torre, A. Tomadin, M. Polini, and A. N. Grigorenko. Nano Lett. 2018, 18, 282.

[143] J. D. Cox and F. J. García de Abajo. Optica 2018, 5, 429.

[144] J. D. Cox and F. J. García de Abajo. Phys. Rev. Lett. 2018, 121, 257403.

[145] I. A. Calafell, J. D. Cox, M. Radonjić, J. R. M. Saavedra, F. J. García de Abajo, L. A. Rozema, and P. Walther. npj Quantum Inf. 2019, 5, 37.

[146] T. Jiang, V. Kravtsov, M. Tokman, A. Belyanin, and M. B. Raschke. Nat. Nanotechnol. 2019, 14, 838.

[147] J. D. Cox and F. J. García de Abajo. Nano Lett. 2019, 19, 3743.

[148] C. Wolff, C. Tserkezis, and N. A. Mortensen. New J. Phys. 2019, 21, 073046.

[149] J. D. Cox and F. J. García de Abajo. Acc. Chem. Res. 2019, $52,2536$.

[150] S. A. Mikhailov. Europhys. Lett. 2007, 79, 27002.

[151] K. L. Ishikawa. Phys. Rev. B 2010, 82, 201402(R).

[152] E. Hendry, P. J. Hale, J. Moger, A. K. Savchenko, and S. A. Mikhailov. Phys. Rev. Lett. 2010, 105, 097401.

[153] A. Y. Bykov, T. V. Murzina, M. G. Rybin, and E. D. Obraztsova. Phys. Rev. B 2012, 85, 121413(R).

[154] H. Zhang, S. Virally, Q. Bao, L. K. Ping, S. Massar, N. Godbout, and P. Kockaert. Opt. Lett. 2012, 37, 1856.
[155] T. Gu, N. Petrone, J. F. McMillan, A. van der Zande, M. Yu, G. Q. Lo, D. L. Kwong, J. Hone, and C. W. Wong. Nat. Photon. 2012, 6, 554.

[156] N. Kumar, J. Kumar, C. Gerstenkorn, R. Wang, H.-Y. Chiu, A. L. Smirl, and H. Zhao. Phys. Rev. B 2013, 87, 121406(R).

[157] S.-Y. Hong, J. I. Dadap, N. Petrone, P.-C. Yeh, J. Hone, and R. M. Osgood, Jr. Phys. Rev. X 2013, 3, 021014.

[158] Y. Q. An, J. E. Rowe, D. B. Dougherty, J. U. Lee, and A. C. Diebold. Phys. Rev. B 2014, 89, 115310.

[159] T. Jiang, D. Huang, J. Cheng, X. Fan, Z. Zhang, Y. Shan, Y. Yi, Y. Dai, L. Shi, K. Liu, C. Zeng, J. Zi, J. E. Sipe, Y.-R. Shen, W.-T. Liu, and S. Wu. Nat. Photonics 2018, 12, 430.

[160] G. Soavi, G. Wang, H. Rostami, D. G. Purdie, D. D. Fazio, T. Ma, B. Luo, J. Wang, A. K. Ott, D. Yoon, S. A. Bourelle, J. E. Muench, I. Goykhman, S. D. Conte, M. Celebrano, A. Tomadin, M. Polini, G. Cerullo, and A. C. Ferrari. Nat. Nanotechnol. 2018, 13, 583.

[161] J. L. Cheng, N. Vermeulen, and J. E. Sipe. New J. Phys. 2014, 16, 053104.

[162] H. A. Hafez, S. Kovalev, J.-C. Deinert, Z. Mics, B. Green, N. Awari, M. Chen, S. Germanskiy, U. Lehnert, J. Teichert, Z. Wang, K.-J. Tielrooij, Z. Liu, Z. Chen, A. Narita, K. Müllen, M. Bonn, M. Gensch, and D. Turchinovich. Nature 2018, 561, 507.

[163] N. Yoshikawa, T. Tamaya, and K. Tanaka. Science 2017, 356,736

[164] M. Baudisch, A. Marini, J. D. Cox, T. Zhu, F. Silva, S. Teichmann, M. Massicotte, F. Koppens, L. S. Levitov, F. J. García de Abajo, and J. Biegert. Nat. Commun. 2018, 9, 1018.

[165] N. M. R. Peres, Y. V. Bludov, J. E. Santos, A.-P. Jauho, and M. I. Vasilevskiy. Phys. Rev. B 2014, 90, 125425.

[166] J. L. Cheng, N. Vermeulen, and J. E. Sipe. Phys. Rev. B 2015, 91, 235320.

[167] S. A. Mikhailov. Phys. Rev. B 2016, 93, 085403.

[168] A. Marini, J. D. Cox, and F. J. García de Abajo. Phys. Rev. B 2017, 95, 125408.

[169] G. X. Ni, L. Wang, M. D. Goldflam, M. Wagner, Z. Fei, A. S. McLeod, M. K. Liu, F. Keilmann, B. Özyilmaz, A. H. Castro Neto, J. Hone, M. M. Fogler, and D. N. Basov. Nat. Photonics 2016, 10, 244.

[170] A. Lauchner, A. Schlather, A. Manjavacas, Y. Cui, M. J. McClain, G. J. Stec, F. J. García de Abajo, P. Nordlander, and N. J. Halas. Nano Lett. 2015, 15, 6208.

[171] K. Müllen. ACS Nano 2014, 8, 6531.

[172] P. Ruffieux, S. Wang, B. Yang, C. Sánchez-Sánchez, J. Liu, T. Dienel, L. Talirz, P. Shinde, C. A. Pignedoli, D. Passerone, T. Dumslaff, X. Feng, K. Müllen, and R. Fasel. Nature 2016, $531,489$.

[173] P. Lodahl, S. Mahmoodian, and S. Stobbe. Rev. Mod. Phys. 2015, 87, 347.

[174] X.-L. Chu, S. Götzinger, and V. Sandoghdar. Nat. Photonics 2017, 11, 58.

[175] J.-M. Raimond, M. Brune, and S. Haroche. Rev. Mod. Phys. 2001, 73, 565.

[176] M. Pelton. Nat. Photonics 2015, 9, 427.

[177] K. J. Russell, T.-L. Liu, S. Cui, and E. L. Hu. Nat. Photonics 2012, 6, 459 .

[178] G. M. Akselrod, C. Argyropoulos, T. B. Hoang, C. Ciracì, C. Fang, J. Huang, D. R. Smith, and M. H. Mikkelsen. Nat. Photonics 2014, 8, 835.

[179] I. M. Palstra, H. M. Doeleman, and A. F. Koenderink. Nanophotonics 2019, 8, 1513. 
[180] G. Khitrova, H. M. Gibbs, M. Kira, S. W. Koch, and A. Scherer. Nature Phys. 2006, $2,81$.

[181] I. Carusotto and C. Ciuti. Rev. Mod. Phys. 2013, 85, 299.

[182] D. E. Chang, A. S. Sørensen, E. A. Demler, and M. D. Lukin. Nature Phys. 2007, 3, 807.

[183] A. Wallraff, D. I. Schuster, A. Blais, L. Frunzio, R.S. Huang, J. Majer, S. Kumar, S. M. Girvin, and R. J. Schoelkopf. Nature 2004, 431, 162.

[184] D. Sanvitto and S. Kéna-Cohen. Nat. Mater. 2016, 15, 1061.

[185] Y. Kaluzny, P. Goy, M. Gross, J. Raimond, and S. Haroche. Phys. Rev. Lett. 1983, 51, 1175.

[186] C. Weisbuch, M. Nishioka, A. Ishikawa, and Y. Arakawa. Phys. Rev. Lett. 1992, 69, 3314.

[187] D. G. Lidzey, D. Bradley, M. Skolnick, T. Virgili, S. Walker, and D. Whittaker. Nature 1998, 395, 53.

[188] J. Bellessa, C. Bonnand, J. C. Plenet, and J. Mugnier. Phys. Rev. Lett. 2004, 93, 036404.

[189] S. Kéna-Cohen and S. Forrest. Nat. Photonics 2010, 4, 371.

[190] E. Orgiu, J. George, J. Hutchison, E. Devaux, J. Dayen, B. Doudin, F. Stellacci, C. Genet, J. Schachenmayer, C. Genes, G. Pupillo, P. Samorì, and T. W. Ebbesen. Nat. Mater. 2015, 14, 1123.

[191] A. Krasnok, S. Lepeshov, and A. Alú. Opt. Express 2018, 26, 15972

[192] P. A. D. Gonçalves, L. P. Bertelsen, S. Xiao, and N. A. Mortensen. Phys. Rev. B 2018, 97, 041402(R).

[193] J. Wen, H. Wang, W. Wang, Z. Deng, C. Zhuang, Y. Zhang, F. Liu, J. She, J. Chen, H. Chen, S. Deng, and N. Xu. Nano Lett. 2017, 17, 4689.

[194] D. Zheng, S. Zhang, Q. Deng, M. Kang, P. Nordlander, and H. Xu. Nano Lett. 2017, 17, 3809.

[195] D. G. Baranov, M. Wersall, J. Cuadra, T. J. Antosiewicz, and T. Shegai. ACS Photonics 2017, 5, 24.

[196] M.-E. Kleemann, R. Chikkaraddy, E. M. Alexeev, D. Kos, C. Carnegie, W. Deacon, A. C. de Pury, C. Große, B. de Nijs, J. Mertens, A. I. Tartakovskii, and J. J. Baumberg. Nat. Commun. 2017, 8, 1296.

[197] J. Cuadra, D. G. Baranov, M. Wersall, R. Verre, T. J. Antosiewicz, and T. Shegai. Nano Lett. 2018, 18, 1777.

[198] M. Stührenberg, B. Munkhbat, D. G. Baranov, J. Cuadra, A. B. Yankovich, T. J. Antosiewicz, E. Olsson, and T. Shegai. Nano Lett. 2018, 18, 5938.

[199] M. Geisler, X. Cui, J. Wang, T. Rindzevicius, L. Gammelgaard, B. S. Jessen, P. Gonçalves, F. Todisco, P. Bøggild, A. Boisen, M. Wubs, N. A. Mortensen, S. Xiao, and N. Stenger. ACS Photonics 2019, 6, 994.

[200] A. B. Yankovich, B. Munkhbat, D. G. Baranov, J. Cuadra, E. Olsén, H. Lourenço-Martins, L. H. Tizei, M. Kociak, E. Olsson, and T. Shegai. arXiv:1905.04067 2019,

[201] B. Chakraborty, J. Gu, Z. Sun, M. Khatoniar, R. Bushati, A. L. Boehmke, R. Koots, and V. M. Menon. Nano Lett. 2018, 18, 6455.

[202] S. Wang, S. Li, T. Chervy, A. Shalabney, S. Azzini, E. Orgiu, J. A. Hutchison, C. Genet, P. Samorì, and T. W. Ebbesen. Nano Lett. 2016, 16, 4368.

[203] H. Leng, B. Szychowski, M.-C. Daniel, and M. Pelton. Nat. Commun. 2018, 9, 4012.

[204] K. Santhosh, O. Bitton, L. Chuntonov, and G. Haran. Nat. Commun. 2016, 7, 11823.

[205] H. Groß, J. M. Hamm, T. Tufarelli, O. Hess, and B. Hecht. Science Adv. 2018, 4, eaar4906.
[206] K.-D. Park, M. A. May, H. Leng, J. Wang, J. A. Kropp, T. Gougousi, M. Pelton, and M. B. Raschke. Sci. Adv. 2019, 5, eaav5931.

[207] R. Chikkaraddy, B. De Nijs, F. Benz, S. J. Barrow, O. A. Scherman, E. Rosta, A. Demetriadou, P. Fox, O. Hess, and J. J. Baumberg. Nature 2016, 535, 127.

[208] A. E. Schlather, N. Large, A. S. Urban, P. Nordlander, and N. J. Halas. Nano Lett. 2013, 13, 3281.

[209] A. I. Vkevinen, R. J. Moerland, H. T. Rekola, A.-P. Eskelinen, J.-P. Martikainen, D.-H. Kim, and P. Trm. Nano Lett. 2014, 14, 1721.

[210] G. Zengin, M. Wersäll, S. Nilsson, T. J. Antosiewicz, M. Käll, and T. Shegai. Phys. Rev. Lett. 2015, 114, 157401.

[211] M. Wersall, J. Cuadra, T. J. Antosiewicz, S. Balci, and T. Shegai. Nano Lett. 2016, 17, 551.

[212] J. T. Hugall, A. Singh, and N. F. van Hulst. ACS Photonics 2018, 5, 43.

[213] F. Marquier, C. Sauvan, and J.-J. Greffet. ACS Photonics 2017, 4, 2091.

[214] P. Vasa and C. Lienau. ACS Photonics 2017, 5, 2.

[215] P. Törmä and W. L. Barnes. Rep. Prog. Phys. 2014, 78, 013901.

[216] X. Zhang, C. De-Eknamkul, J. Gu, A. L. Boehmke, V. M. Menon, J. Khurgin, and E. Cubukcu. Nat. Nanotechnol. 2019, $14,844$.

[217] S. Hou, L. Y. Tobing, X. Wang, Z. Xie, J. Yu, J. Zhou, D. Zhang, C. Dang, P. Coquet, B. K. Tay, M. D. Birowosuto, E. H. T. Teo, and H. Wang. Adv. Opt. Mater. 2019, 7, 1900857.

[218] C. Tserkezis, A. Fernández-Domínguez, P. Gonçalves, F. Todisco, J. Cox, K. Busch, N. Stenger, S. Bozhevolnyi, N. A. Mortensen, and C. Wolff. arXiv:1907.02605 2019, .

[219] M. Pelton, S. D. Storm, and H. Leng. Nanoscale 2019, 11, 14540.

[220] F. Todisco, R. Malureanu, C. Wolff, P. A. D. Gonçalves, A. S. Roberts, N. A. Mortensen, and C. Tserkezis. arXiv:1906.09898 2019,

[221] G. Wang, A. Chernikov, M. M. Glazov, T. F. Heinz, X. Marie, T. Amand, and B. Urbaszek. Rev. Mod. Phys. 2018, 90, 021001.

[222] K. S. Thygesen. 2D Mater. 2017, 4, 022004.

[223] Y. Li, A. Chernikov, X. Zhang, A. Rigosi, H. M. Hill, A. M. van der Zande, D. A. Chenet, E.-M. Shih, J. Hone, and T. F. Heinz. Phys. Rev. B 2014, 90, 205422.

[224] A. Chernikov, T. C. Berkelbach, H. M. Hill, A. Rigosi, Y. Li, O. B. Aslan, D. R. Reichman, M. S. Hybertsen, and T. F. Heinz. Phys. Rev. Lett. 2014, 113, 076802.

[225] A. J. Chaves, R. M. Ribeiro, T. Frederico, and N. M. R. Peres. 2D Mater. 2017, 4, 025086.

[226] D. Xiao, G.-B. Liu, W. Feng, X. Xu, and W. Yao. Phys. Rev. Lett. 2012, 108, 196802.

[227] H. Zeng, J. Dai, W. Yao, D. Xiao, and X. Cui. Nat. Nanotechnol. 2012, 7, 490.

[228] T. Cao, G. Wang, W. Han, H. Ye, C. Zhu, J. Shi, Q. Niu, P. Tan, E. Wang, B. Liu, and J. Feing. Nat. Commun. 2012, $3,887$.

[229] A. M. Jones, H. Yu, N. J. Ghimire, S. Wu, G. Aivazian, J. S. Ross, B. Zhao, J. Yan, D. G. Mandrus, D. Xiao, W. Yao, and X. X. Nat. Nanotechnol. 2013, 8, 634.

[230] S.-H. Gong, F. Alpeggiani, B. Sciacca, E. C. Garnett, and L. Kuipers. Science 2018, 359, 443. 
[231] T. Chervy, S. Azzini, E. Lorchat, S. Wang, Y. Gorodetski, J. A. Hutchison, S. Berciaud, T. W. Ebbesen, and C. Genet. ACS Photonics 2018, 5, 1281.

[232] J. S. Ross, P. Klement, A. M. Jones, N. J. Ghimire, J. Yan, D. Mandrus, T. Taniguchi, K. Watanabe, K. Kitamura, W. Yao, D. H. Cobden, and X. Xu. Nat. Nanotechnol. 2014, 9, 268.

[233] Y. You, X.-X. Zhang, T. C. Berkelbach, M. S. Hybertsen, D. R. Reichman, and T. F. Heinz. Nature Phys. 2015, 11, 477.

[234] A. Steinhoff, M. Florian, A. Singh, K. Tran, M. Kolarczik, S. Helmrich, A. W. Achtstein, U. Woggon, N. Owschimikow, F. Jahnke, and X. Li. Nat. Phys. 2018, 14,1199

[235] J. Pei, J. Yang, X. Wang, F. Wang, S. Mokkapati, T. Lu, J.-C. Zheng, Q. Qin, D. Neshev, H. H. Tan, C. Jagadish, and Y. Lu. ACS Nano 2017, 11, 7468.

[236] A. Almand-Hunter, H. Li, S. Cundiff, M. Mootz, M. Kira, and S. W. Koch. Nature 2014, 506, 471.

[237] H. Deng, H. Haug, and Y. Yamamoto. Rev. Mod. Phys. 2010, 82, 1489.

[238] A. Shivayogimath, J. D. Thomsen, D. M. Mackenzie, M. Geisler, R.-M. Stan, A. J. Holt, M. Bianchi, A. Crovetto, P. R. Whelan, A. Carvalho, A. H. Castro Neto, P. Hofmann, S. Stenger, P. Bøggild, and T. J. Booth. Nat. Commun. 2019, 10, 2957.

[239] A. F. Kockum, A. Miranowicz, S. D. Liberato, S. Savasta, and F. Nori. Nat. Rev. Phys. 2019, 1, 19.

[240] A. M. Dibos, Y. Zhou, L. A. Jauregui, G. Scuri, D. S. Wild, A. A. High, T. Taniguchi, K. Watanabe, M. D. Lukin, P. Kim, and H. Park. Nano Lett. 2019, 19, 3543.

[241] L. C. Flatten, Z. He, D. M. Coles, A. A. P. Trichet, A. W. Powell, R. A. Taylor, J. H. Warner, and J. M. Smith. Sci. Rep. 2016, 6.

[242] X. Han, K. Wang, X. Xing, M. Wang, and P. Lu. ACS Photonics 2018, 5, 3970.

[243] X. Chen, H. Wang, N.-S. Xu, H. Chen, and S. Deng. App. Mater. Today 2019, 15, 145.

[244] B. Lee, J. Park, G. H. Han, H.-S. Ee, C. H. Naylor, W. Liu, A. C. Johnson, and R. Agarwal. Nano Lett. 2015, 15, 3646.

[245] W. Liu, B. Lee, C. H. Naylor, H.-S. Ee, J. Park, A. T. C. Johnson, and R. Agarwal. Nano Lett. 2016, 16, 1262.

[246] B. Lee, W. Liu, C. H. Naylor, J. Park, S. C. Malek, J. S. Berger, A. T. C. Johnson, and R. Agarwal. Nano Lett. 2017, 17,4541

[247] X. Lu, M. Rycenga, S. E. Skrabalak, B. Wiley, and Y. Xia. Annu. Rev. Phys. Chem. 2009, 60, 167.

[248] F. Qin, T. Zhao, R. Jiang, N. Jiang, Q. Ruan, J. Wang, L.-D. Sun, C.-H. Yan, and H.-Q. Lin. Adv. Opt. Mater. 2016, 4, 76.

[249] H. Zeng, G.-B. Liu, J. Dai, Y. Yan, B. Zhu, R. He, L. Xie, S. Xu, X. Chen, W. Yao, and X. Cui. Sci. Rep. 2013, 3, 1608.

[250] T. J. Antosiewicz, S. P. Apell, and T. Shegai. ACS Photonics 2014, 1,454

[251] M. Wersäll, B. Munkhbat, D. G. Baranov, F. Herrera, J. Cao, T. J. Antosiewicz, and T. Shegai. ACS Photonics 2019, 6, 2570.

[252] L. Liu, L. Y. Tobing, X. Yu, J. Tong, B. Qiang, A. I. Fernández-Domínguez, F. J. Garcia-Vidal, D. H. Zhang, Q. J. Wang, and Y. Luo. Adv. Opt. Mater. 2019, 7, 1901002.
[253] K. Tran, G. Moody, F. Wu, X. Lu, J. Choi, K. Kim, A. Rai, D. A. Sanchez, J. Quan, A. Singh, J. Embley, A. Zepeda, M. Campbell, T. Autry, T. Taniguchi, K. Watanabe, N. Lu, S. K. Banerjee, K. L. Silverman, S. Kim, E. Tutuc, L. Yang, A. H. MacDonald, and X. Li. Nature 2019, 567, 71.

[254] K. L. Seyler, P. Rivera, H. Yu, N. P. Wilson, E. L. Ray, D. G. Mandrus, J. Yan, W. Yao, and X. Xu. Nature 2019, 567, 66.

[255] C. Jin, E. C. Regan, A. Yan, M. I. B. Utama, D. Wang, S. Zhao, Y. Qin, S. Yang, Z. Zheng, S. Shi, K. Watanabe, T. Taniguchi, S. Tongay, A. Zettl, and F. Wang. Nature 2019, 567,76

[256] E. M. Alexeev, D. A. Ruiz-Tijerina, M. Danovich, M. J. Hamer, D. J. Terry, P. K. Nayak, S. Ahn, S. Pak, J. Lee, J. I. Sohn, M. R. Molas, M. Koperski, K. Watanabe, T. Taniguchi, K. S. Novoselov, R. V. Gorbachev, H. S. Shin, V. I. Fal'ko, and A. I. Tartakovskii. Nature 2019, 567, 81.

[257] P. Vasa, W. Wang, R. Pomraenke, M. Lammers, M. Maiuri, C. Manzoni, G. Cerullo, and C. Lienau. Nat. Photonics 2013, 7, 128 .

[258] A. I. Fernández-Domínguez, S. I. Bozhevolnyi, and N. A. Mortensen. ACS Photonics 2018, 5, 3447.

[259] R. Sáez-Blázquez, J. Feist, A. Fernández-Domínguez, and F. García-Vidal. Optica 2017, 4, 1363.

[260] O. S. Ojambati, R. Chikkaraddy, W. D. Deacon, M. Horton, D. Kos, V. A. Turek, U. F. Keyser, and J. J. Baumberg. Nat. Commun. 2019, 10, 1049.

[261] A. Nemilentsau, T. Low, and G. Hanson. Phys. Rev. Lett. 2016, 116, 066804.

[262] J. C. W. Song and M. S. Rudner. Proc. Natl. Acad. Sci. U. S. A. 2016, $113,4658$.

[263] N. Rivera, I. Kaminer, B. Zhen, J. D. Joannopoulos, and M. Soljačić. Science 2016, 353, 263.

[264] L. Ju, Z. Shi, N. Nair, Y. Lv, C. Jin, J. Velasco, C. OjedaAristizabal, H. A. Bechtel, M. C. Martin, A. Zettl, J. Analytis, and F. Wang. Nature 2015, 520, 650.

[265] A. B. Khanikaev and G. Shvets. Nat. Photonics 2017, 11, 763.

[266] F. Hu, S. R. Das, Y. Luan, T.-F. Chung, Y. P. Chen, and Z. Fei. Phys. Rev. Lett. 2017, 119, 247402.

[267] C. Lewandowski and L. Levitov. Proc. Natl. Acad. Sci. U. S. A. 2019, 116, 20869.

[268] N. C. Hesp, I. Torre, D. Rodan-Legrain, P. Novelli, Y. Cao, S. Carr, S. Fang, P. Stepanov, D. Barcons-Ruiz, H. HerzigSheinfux, K. Watanabe, T. Taniguchi, D. K. Efetov, E. Kaxiras, P. Jarillo-Herrero, M. Polini, and F. H. Koppens. arXiv 2019, arXiv:1910.07893.

[269] A. Boltasseva and V. M. Shalaev. ACS Photonics 2019, 6, 1.

[270] D. K. Efetov and P. Kim. Phys. Rev. Lett. 2010, 105, 256805. 